\title{
Genome-wide copy number analysis of circulating tumor cells in breast cancer patients with liver metastasis
}

\author{
LINGLIN ZOU $^{1 *}$, SABER IMANI $^{*}$, MAZAHER MAGHSOUDLOO $^{2,3}$, MARZIEH DEHGHAN SHASALTANEH $^{4}$, \\ LANYANG GAO $^{5}$, JIA ZHOU ${ }^{6}$, QINGLIAN WEN ${ }^{1}$, SHUYA LIU ${ }^{1}$, LEISHENG ZHANG ${ }^{7}$ and GANG CHEN ${ }^{8}$ \\ ${ }^{1}$ Department of Oncology, The Affiliated Hospital of Southwest Medical University, Southwest Medical University, Luzhou, \\ Sichuan 646000, P.R. China; ${ }^{2}$ Laboratory of Systems Biology and Bioinformatics (LBB), Institute of Biochemistry and \\ Biophysics, University of Tehran, Tehran 1417614411; ${ }^{3}$ Department of Genetics, Faculty of Advanced Science and Technology, \\ Tehran Medical Sciences, Islamic Azad University, Tehran 1916893813; ${ }^{4}$ Department of Biology, Faculty of Science, \\ University of Zanjan, Zanjan 4537138791, Iran; ${ }^{5}$ Sichuan Provincial Center for Gynaecology and Breast Disease, The \\ Affiliated Hospital of Southwest Medical University; ${ }^{6}$ School of Humanities and Management Science, Southwest Medical \\ University, Luzhou, Sichuan 646000; ${ }^{7}$ The Postdoctoral Research Station, School of Medicine, Nankai University, \\ Tianjin 300071; ${ }^{8}$ Department of Medical Equipment, The Affiliated Hospital of Southwest Medical University, \\ Southwest Medical University, Luzhou, Sichuan 646000, P.R. China
}

Received December 6, 2019; Accepted May 12, 2020

DOI: $10.3892 /$ or.2020.7650

\begin{abstract}
Correspondence to: Professor Leisheng Zhang, The Postdoctoral Research Station, School of Medicine, Nankai University, 94 Weijin Road, Tianjin 300071, P.R. China

E-mail: leisheng_zhang@163.com

Professor Gang Chen, Department of Medical Equipment, The Affiliated Hospital of Southwest Medical University, Southwest Medical University, 25 Taiping Street, Jiangyang, Luzhou, Sichuan 646000, P.R. China

E-mail: chengang@swmu.edu.cn

${ }^{*}$ Contributed equally
\end{abstract}

Abbreviations: BCLM, breast cancer liver metastasis; MBC, metastatic breast cancer; CT, computed tomography; MRI, magnetic resonance imaging; PET, positron emission tomography; CNVs, copy number variations; WGS, whole genome sequencing; CTCs, circulating tumor cells; ${ }^{18} \mathrm{~F}$-FDG PET/CT, fluorine-18-fluorodeoxyglucose positron emission tomography/computed tomography; CK, cytokeratin; MALBACs, multiple annealing and looping based amplification cycles; AST, aspartate aminotransferase; ALT, alanine aminotransferase; ALP, alkaline phosphatase; PT, prothrombin time; EPCs, epithelial cells; gDNA, genomic DNA; SD, standard deviation; WGA, whole genome amplification; H\&E, hematoxylin and eosin; SUV, standardized uptake value; HMMs, Hidden Markov Models; SUVmax, maximum-pixel SUV; qPCR, quantitative PCR; GSEA, gene set enrichment analysis; hBDs, human $\beta$-defensins; PPI, protein-protein interaction

Key words: breast cancer liver metastasis, circulating tumor cells, genome-wide copy number analysis, newly diagnosed liver metastases, recurrent liver metastases
Abstract. The genome-wide copy number analysis of circulating tumor cells (CTCs) provides a promising prognostic biomarker for survival in breast cancer liver metastasis (BCLM) patients. The present study aimed to confirm the prognostic value of the presence of CTCs in BCLM patients. We previously developed an assay for the genome-wide pattern differences in copy number variations (CNVs) as an adjunct test for the routine imaging and histopathologic diagnosis methods to distinguish newly diagnosed liver metastases and recurrent liver metastases. Forty-three breast cancer patients were selected for this study in which 23 newly diagnosed and 20 recurrent liver metastases were diagnosed by histopathology and ${ }^{18} \mathrm{~F}$-FDG PET/CT imaging. CTCs were counted from all patients using the CellSearch system and were confirmed by cytomorphology and three-color immunocytochemistry. Genomic DNA of single CTCs was amplified using multiple annealing and looping based amplification cycles (MALBAC). Then, we compared the CTC numbers of newly diagnosed and recurrent BCLM patients using Illumina platforms. A high CTC frequency (>15 CTCs/7.5 ml blood) was found to be correlated with disease severity and metastatic progression, which suggests the value for CTCs in the diagnosis of BCLM in comparison with pathohistology and PET/CT imaging ( $\mathrm{P}>0.05)$. Moreover, CTCs isolated from BCLM patients remained an independent prognostic detection factor associated with overall survival $(\mathrm{P}=0.0041)$. Comparison between newly diagnosed and recurrent liver metastases revealed different frequencies of CNVs $(\mathrm{P}>0.05)$. Notably, the CNV pattern of isolated CTCs of recurrent BCLM patients was similar to recurrent liver metastases (nearly $82 \%$ of the gain/loss regions). Functional enrichment analysis identified 25 genes as a CNV signature of BCLM. Among them, were defensin and $\beta$-defensin genes, which are significantly associated with anti-angiogenesis and immunomodulation signaling pathways. High CTC frequencies are effective in the 
evaluation and differentiation between newly diagnosed liver metastases from recurrent liver metastases. Future clinical studies will be necessary to fully determine the prognostic potential of CTC cluster signatures in patients with BCLM.

\section{Introduction}

Breast cancer liver metastasis (BCLM) is the most common metastatic event associated with breast cancer, with a median overall survival rate of 4.8-9.2 months and a 5-year survival rate of $23 \%(1,2)$. Nearly $40-50 \%$ of women with metastatic breast cancer (MBC) are diagnosed with liver metastasis (3). Current treatments for BCLM include systemic therapy such as endocrine therapy, targeted therapy, chemotherapy, and local therapy such as radioembolization, chemoembolization, microwave ablation and stereotactic body radiotherapy (4-6). Despite routine comprehensive treatments, BCLM is still incurable and carries a poor prognosis, especially for patients who exhibit poor response to chemotherapy or who have estrogen receptor (ER)-negative disease. Therefore, accurate diagnosis of BCLM is considerably important for improving the prognosis of BCLM. In addition, the ability to distinguish between newly diagnosed and recurrent BCLM has significant diagnostic and prognostic value $(7,8)$. Current diagnostic methods for BCLM are mostly based on abnormal liver function tests, imaging examination such as ultrasound, computed tomography (CT), and magnetic resonance imaging (MRI). These methods have limitations in distinguishing between newly diagnosed and recurrent BCLM, as recurrent metastatic foci have distinct morphology and a large number of genomic alterations, neither of which are detected by the above diagnostic methods. To ensure early site-specific BCLM detection as well as distinguish between newly diagnosed and recurrent BCLM, metastatic niche cells must be characterized by alterations in gene expression (9), deposition of tissue homeostasis $(10,11)$, and infiltration of numerous immune cell populations $(12,13)$.

Genome sequencing-based molecular analysis has proven to be a strong approach for the diagnosis of heterogeneous monogenetic BCLM, with the ability to characterize copy number variations (CNVs), loss of heterozygosity, and analyze somatic mutations (14-16). Profiling of CNVs has been an accretive and reliable analytical tool in distinguishing between newly diagnosed and recurrent metastases and uses an ultra-low input of blood- or tissue-derived DNA (15). In comparison with traditional BCLM detection methods, measuring genome-wide CNVs is a more sensitive and cost-effective analytical method that detects the CNV profile without depleting the sample resource $(5,17)$. Furthermore, this type of molecular analysis has been proven to be a prospective approach for the clinical diagnosis of other heterogeneous monogenetic types of $\mathrm{MBC}$, and is a step toward genetic counseling and potential gene replacement therapy $(5,18)$.

It is well established that enumeration and monitoring of circulating tumor cells (CTCs) are useful for the diagnosis and prognostic prediction of many cancer types, such as breast (19-21), prostate (22), and lung cancer (23). CTCs are routinely detectable in the blood stream of cancer patients with both early and late stage cancer (20). Although the analysis of CTCs requires significant technical skill, laboratory resources, and instrumentation, it is an assay that is frequently established in specialized medical centers. Notably, the genome-wide copy number analysis of CTCs provides a promising diagnostic and prognostic biomarker for survival in metastatic patients. The molecular characterization and monitoring of CTCs by utilizing high-sensitivity and high-throughput technologies guarantees a promising platform for capturing and determining the organotropism of metastatic niche cells (20).

Many recent studies have attempted to determine whether genome-wide CNVs of CTCs may be a diagnostic and prognostic factor for survival in breast cancer patients $(20,21,24)$. However, studies on the clinical efficiency of whole genome sequencing (WGS) for profiling CNVs are often challenging and their findings, contentious. In addition, only a few investigations have proposed that CNVs of CTCs can help to distinguish between newly diagnosed and recurrent metastases (25).

Here, for the first time, we conducted a prospective clinical investigation to confirm the diagnostic value of genome-wide $\mathrm{CNVs}$ in BCLM. We compared the consistency and efficiency of genome-wide CNVs with other comment methods used for the detection of BCLM. Furthermore, we developed a higher efficiency WGS technique using CNV profiling to distinguish between newly diagnosed and recurrent BCLM. This may provide a potentially valuable approach for the genetic characterization of BCLM.

\section{Materials and methods}

Patient population and clinical assessment. All patients with invasive ductal carcinoma of the breast were preliminarily selected for this prospective study at the Affiliated Hospital of Southwest Medical University, Luzhou, Sichuan from March 2019 to December 2019. Totally 43 patients were selected according to the inclusion criteria and subdivided. The mean age of the patients was 49.87 years (range, 39-57 years). The patients were suspected to have newly diagnosed or postoperative recurrent liver metastasis as assessed by fluorine-18-fluorodeoxyglucose positron emission tomography/computed tomography $\left({ }^{18} \mathrm{~F}-\mathrm{FDG} \mathrm{PET} / \mathrm{CT}\right)$ imaging. The breast tumors were reviewed and liver metastases were confirmed by two expert pathologists (ZeL and LG). The patients were excluded if the liver nodules were benign lesions, primary liver tumors, or metastases of other organs besides the breast. Next, the demographic information and detailed history of the included patients were documented according to an interviewer-administered questionnaire. Laboratory tests including blood routine, liver function tests [aspartate aminotransferase (AST), alanine aminotransferase (ALT), alkaline phosphatase (ALP), prothrombin time (PT), albumin, and bilirubin] and a ${ }^{18} \mathrm{~F}$-FDG PET/CT were performed. All participants received different systematic and local therapies at the Department of Oncology, The Affiliated Hospital of Southwest Medical University (Luzhou, Sichuan).

Study design. Fig. 1 shows the present study model in detail. Firstly, a suspected malignant liver nodule was identified by ${ }^{18} \mathrm{~F}-\mathrm{FDG}$ PET/CT scan in all patients, which was then confirmed by pathology within a week. Next, CTCs from each selected BCLM patient were captured with the CellSearch platform using antibody enrichment, and were further isolated under a fluorescence microscope with $94 \%$ specificity. Then, genomic DNA (gDNA) of single a CTC was amplified using the multiple 


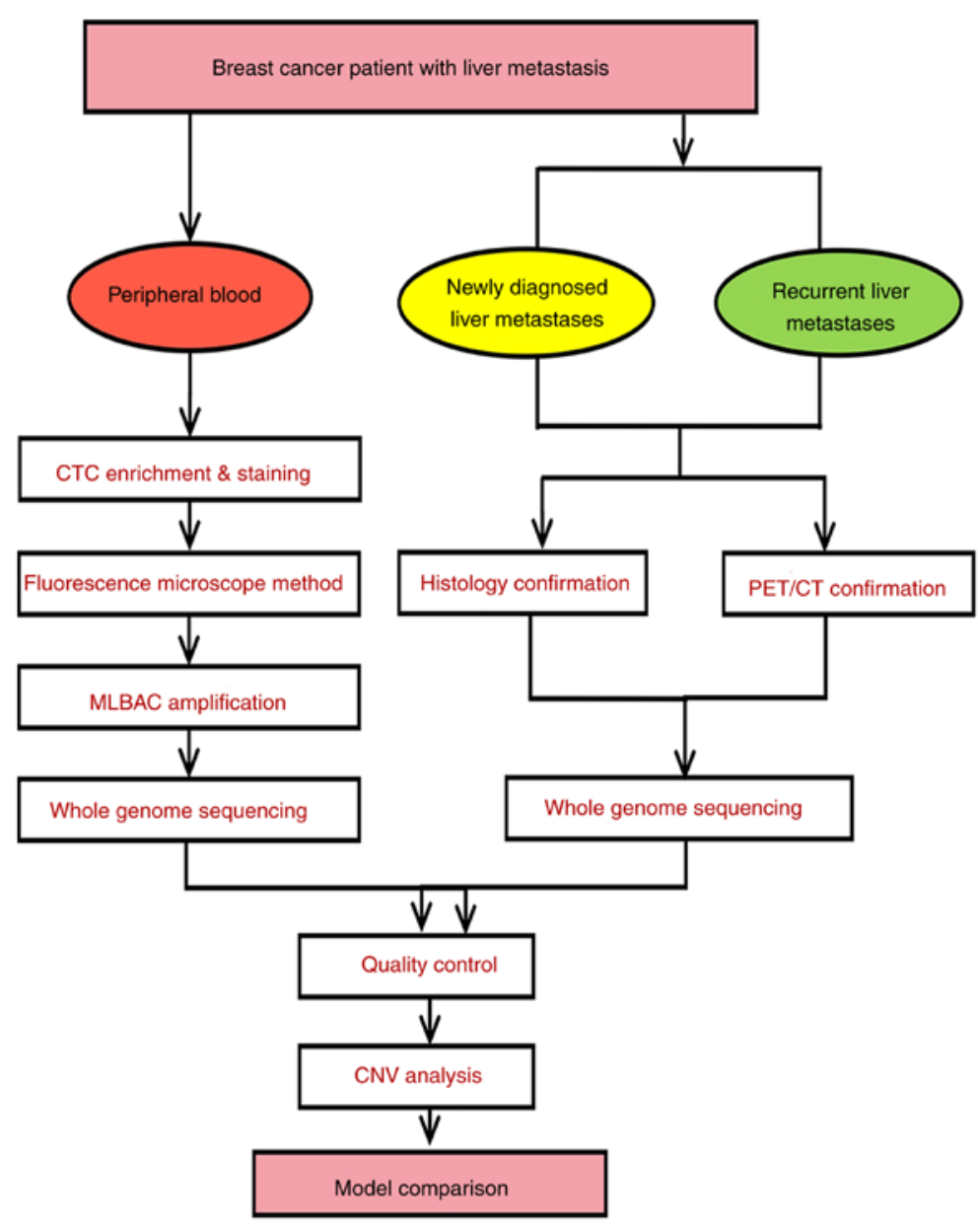

Figure 1. Flow chart of the schematic overview of the current study design for the genome-wide copy number analysis of CTCs of BCLM. CTCs, circulating tumor cells; BCLM, breast cancer liver metastasis; MLBACs, multiple annealing and looping based amplification cycles; CNVs, copy number variations; $\mathrm{PET} / \mathrm{CT}$, positron emission tomography/computed tomography.

annealing and looping based amplification cycle (MALBAC) method. WGS was planned for CTCs as well as newly diagnosed or recurrent liver metastases samples from each CTC positive patient. Furthermore, we analyzed genome-wide CNVs in these cases to introduce the major genomic variations that are specific to and reproducible in BCLM. Finally, noninvasive CTC-based BCLM diagnostics were compared with ${ }^{18} \mathrm{~F}-\mathrm{FDG}$ $\mathrm{PET} / \mathrm{CT}$ and histological findings.

Sample preparation. All tissue samples were fixed and embedded in Tissue Tek II OCT (Miles Scientific), frozen $15 \mathrm{~min}$ in isopentane, precooled in liquid nitrogen, and then stored at $-80^{\circ} \mathrm{C}$ for future pathology assays. Next, the best frozen sample was oriented and cut as 5- $\mu$ m-thick cryostat sections for hematoxylin and eosin (H\&E) analysis and histological conformation. Microscopic analysis of all slides was performed using light microscopy (Olympus Corp.) linked to a computerized imaging system (Image-Pro Plus V6.0; Media Cybernetics, Inc.). The cases were coded and measurements were made in a blinded manner by two expert pathologists (LiZ and SI). Subsequently, from each patient, $7.5 \mathrm{ml}$ fresh blood was collected in a Cell Save blood collection tube (Immunicon Inc.) and stored at $15-20^{\circ} \mathrm{C}$ for CTC enumeration within 3 days after collection and $10 \mathrm{ml}$ was collected for gDNA extraction. The gDNA was extracted from fresh peripheral blood leukocytes, using the Qiagen DNA extraction kit (Qiagen). Primary tumor and liver metastasis tissues were obtained by standard core needle methods.

${ }^{18}$ F-FDG PET/CT imaging. ${ }^{18} \mathrm{~F}-\mathrm{FDG}$ PET/CT imaging was provided by the Department of Nuclear Medicine, The Affiliated Hospital of Southwest Medical University, Luzhou, Sichuan, China. Before scanning, all patients had fasted for at least $6 \mathrm{~h}$ and their blood glucose levels were measured to be within the normal range prior to intravenous injection of $370 \mathrm{MBq}$ of ${ }^{18} \mathrm{~F}-\mathrm{FDG}$. The PET/CT parameters were $120 \mathrm{KV}$, $80 \mathrm{mAs}, 3 \mathrm{~min} / \mathrm{bed}, 0.813 \mathrm{pitch}$, and a $3.8 \mathrm{~mm}$ reconstruction of layer thickness. Immediately after CT scanning, a PET emission scan that covered the identical transverse field of view was acquired in 3-min acquisition time per bed position. Data were reconstructed using an iterative reconstruction technique and attenuation correction derived from the CT data. The CT, PET and fused PET/CT images were transmitted to an Extended Brilliance Philips workstation version 4.1 (Philips Healthcare). The PET images were evaluated qualitatively for regions of focally increased metabolism. An appreciably increased uptake level in comparison to surrounding tissue was considered as malignant lesions. Semi-quantitative analysis using the standardized uptake value (SUV) and the mean \pm SD of maximum-pixel SUV (SUVmax) of the lesions was calculated. 
All the PET/CT scans were evaluated by the unit of nuclear radiologists and two nuclear radiologists (SI and QW).

CTC isolation and capture. CTCs from $7.5 \mathrm{ml}$ blood sample were captured with the CellSearch Circulating Tumor Cell Kit (cat. no. K062013; Veridex, LLC) for $1 \mathrm{~h}$ at room temperature. This kit is a high-throughput isolation kit that is mostly used for enumeration and isolation of CTCs using magnetic beads conjugated to anti-epithelial cell adhesion molecule (EpCAM) antibodies $(26,27)$. EpCAM-positive cells were immediately isolated using a magnetic field. To distinguish cancer cells from leukocytes, $1 \mathrm{ml}$ PBS and isolated CTCs were stained with $10 \mu \mathrm{l}$ DAPI for $15 \mathrm{~min}$ in the dark, $200 \mu \mathrm{l}$ anti-CK-8 (8)-phycoerythrin antibodies for $30 \mathrm{~min}$ and $20 \mu \mathrm{l}$ anti-CD45-allophycocyanin antibodies for $60 \mathrm{~min}$. The cancer cells stained $\mathrm{DAPI}^{+}$, anti-CD45 ${ }^{-}$, and $\mathrm{CK}-8^{+}$, while leukocytes stained DAPI ${ }^{+}$, anti-CD45 ${ }^{+}$, and anti-CK-8- Following immunostaining, the enriched CTCs were re-suspended with PBS and were then manually counted under a fluorescence microscope (10x lens, Axio Imager A2; Carl Zeiss). We obtained full coverage of all the stained CTCs using micropipetting within 5 min. For high-ratio CTC enumeration, UV-exposed water was used to wash the isolated single CTCs repeatedly to minimize DNA contamination. The isolated single CTCs were used for further genome amplification. The identification of all CTCs was performed by an expert pathologist who is specialized in the pathological diagnosis of metastasis (LiZ, SI and QW).

Whole genome amplification of single CTCs. Whole genome amplification (WGA) of an isolated single CTC was performed using MALBAC Single Cell Whole genome amplification Kit (cat. no. YK001A; Yikon Genomics, Inc.). The amplified DNA was purified by the DNA clean-up kit (cat. no. CW2301; CWbio) and the fragment size generated by WGA was between 300-2,000 bp, as determined by gel electrophoresis. Quantitative PCR (qPCR) was performed on seven randomly selected loci in the genome to check the integrity of amplification. All seven loci were amplified with reasonable $\mathrm{Ct}$ number and a deep average 'unique mapped of raw'. The 10x WGS analyses were performed in CTC specimens of newly diagnosed BCLM patients with a high CTCs and also in CTC specimens of recurrent BCLM patients with low CTCs. CTCs were regularly amplified with an average amplified gDNA of $900 \mathrm{ng} / \mathrm{cell}$. Then, we applied 30x WGS sequencing of liver metastases to both newly diagnosed and recurrent BCLM.

Whole genome library preparation and sequencing. The design of whole genome library and sequencing were detected using Illumina paired-end libraries (Illumina, Inc.) methods as previously described (28). Briefly, $900 \mathrm{ng}$ to $1 \mu \mathrm{g}$ of extracted DNA from the amplification products of CTCs by MALBAC or from tumor tissues was sheared into fragments of approximately 300 bp using a Covaris E220 system (Covaris Inc.). The adaptor-ligated DNA was prepared, and enrichment q;PCR was performed on an aliquot of adaptor-ligated DNA to complete the adaptor for Illumina PE sequencing. A total of $600 \mathrm{ng}$ of pooled-DNA from four barcoded libraries was used for hybridization and post-hybridization amplification following the manufacturer's protocol (SureSelectXT Target Enrichment System for Illumina Paired-End Sequencing Library; Illumina,
Inc.). Then, the result of the PCR amplification of single CTCs were quality checked and WGS was performed with the Illumina HiSeq 2000 (Illumina, Inc.). to achieve an average of $\sim 10 \mathrm{x}$ and $\sim 30 \mathrm{x}$ coverage depth, respectively.

Genomic CNVs and gene set enrichment analysis (GSEA). Here, we used the standard probe to determine genomic CNVs in single CTCs that was established by Zong et al (29). In brief, paired-end sequencing reads of each CTC and tumor sample were aligned with the human hg19 reference genome using Burrows-Wheeler Aligner v0.6.1 (30) and the available public online University of Santa Cruz (UCSC) database (http://genome.ucsc.edu/) (30). The Firehose pipeline (level 4) was used to manage input and output files and submit analyses for execution (31). Genome-wide detection of single-nucleotide and CNVs of a single human cell was performed using ControlFreeC (32). A binary array, which indicates whether a single cancer cell has higher coverage than normal leukocytes, was taken as output in Hidden Markov Models-based calling algorithms (HMMs) $(33,34)$. The copy number analysis was performed by applying data on the Ginkgo dataset (http://qb.cshl. edu/ginkgo) and two R packages (HMM copy and DNA copy), with hg19 as the reference genome. Enrichment tests were conducted at the arm level to identify significantly gained and lost chromosome arms. In addition, Gene Set Enrichment Analysis (GSEA) was used for a functional assessment of the recognized disease pathways among different CTC-shared CNVs $(35,36)$. Accordingly, we used pathway analyses to find the potential biological functional assessment of CTC-shared CNVs via R software (v3.3.1) (37,38).

Statistical analysis. According to the CellSearch machine-default, patients with at least five CTCs/7.5 ml were considered CTC-positive. In this study, comparison of group differences was carried out with a one-way analysis of variance (ANOVA) test and then Turkey multiple comparison post-hoc analysis. All statistical analyses were performed using SPSS software v21.0 (IBM, Corp.). All tests were repeated three times or more. Data are presented as means \pm standard deviation (SD) or median (range). A linear regression analysis was carried out to determine independent factors for the diagnosis of CTCs. For data not distributed normally, comparisons between three groups were made using a Kruskal-Wallis one-way analysis of variance, followed by a post-hoc Dunn's test. For all tests, two-sided P-values and adjusted P-values of $<0.05$ were considered statistically significant. All charts were designed using GraphPad Prism v5.0 (GraphPad Software, Inc.).

\section{Results}

Demographic and clinicopathological findings. The demographics and clinicopathological characteristics of the 43 selected patients are detailed in Table I. After considering all exclusion/inclusion criteria, 43 BCLM patients were included in this study. As of now, there is no established cut-off value for a prognostic number of CTCs in BCLM. During this study, we divided our patients by their CTC counts as either lower than, equal to, or higher than the median number of CTCs (5-15 CTCs/7.5 ml blood) to determine any possible correlation with clinicopathological features. Using these criteria, 60\% (26 of 
Table I. Demographic and baseline clinicopathological characteristics of the BCLM patients.

$$
\text { All patients }(\mathrm{N}=43)
$$

CTCs/7.5 ml of blood

Variable

CTC-negative CTC-positive ${ }^{\mathrm{a}} \quad \mathrm{P}_{\text {-value }}{ }^{\mathrm{b}}$

Low-CTCs $(\leq 5)$

High CTCs (>5)

P-value

A, Demographic variables

\begin{tabular}{|c|c|c|c|c|c|c|}
\hline Subjects, n (\%) & $17(39.5)$ & $26(60.5)$ & 0.421 & $14(53.9)$ & $12(46.1)$ & 0.347 \\
\hline Age (years) & $48.51 \pm 3.01$ & $51.23 \pm 3.73$ & 0.278 & $49.27 \pm 3.42$ & $52.05 \pm 2.47$ & 0.491 \\
\hline $\mathrm{BMI}\left(\mathrm{kg} / \mathrm{m}^{2}\right)$ & $23.01 \pm 3.03$ & $21.97 \pm 2.43$ & 0.602 & $22.03 \pm 3.47$ & $22.93 \pm 3.25$ & 0.204 \\
\hline Disease duration (years) & $3.12 \pm 0.97$ & $2.54 \pm 1.01$ & 0.487 & $2.87 \pm 1.07$ & $3.23 \pm 0.93$ & 0.562 \\
\hline PET/CT (SUVmax) & $6.12 \pm 3.44$ & $5.97 \pm 2.82$ & 0.395 & $4.59 \pm 3.27$ & $4.96 \pm 2.73$ & 0.762 \\
\hline
\end{tabular}

B, Histopathology variables, n (\%)

\begin{tabular}{|c|c|c|c|c|c|c|}
\hline Initial disease stage & & & & & & 0.041 \\
\hline II & $3(6.9)$ & $3(6.9)$ & 0.382 & $2(7.6)$ & $1(3.8)$ & \\
\hline III & $6(13.9)$ & $8(18.7)$ & & $4(15.4)$ & $4(15.4)$ & \\
\hline IV & $8(18.7)$ & $15(34.9)$ & & $8(30.8)$ & $7(27)$ & \\
\hline Invasive ductal & $17(39.5)$ & $26(60.5)$ & - & $14(32.6)$ & $12(27.9)$ & - \\
\hline ER status & & & & & & 0.582 \\
\hline Negative & $6(14)$ & $9(20.9)$ & 0.071 & $3(11.5)$ & $6(23.1)$ & \\
\hline Positive & $11(25.6)$ & $17(39.5)$ & & $11(42.3)$ & $6(23.1)$ & \\
\hline PR statue & & & & & & 0.231 \\
\hline Negative & $5(11.6)$ & $9(20.9)$ & 0.542 & $3(11.5)$ & $6(23.1)$ & \\
\hline Positive & $12(28)$ & $17(39.5)$ & & $11(42.3)$ & $6(23.1)$ & \\
\hline HER2 status & & & & & & 0.074 \\
\hline Negative & $10(23.2)$ & $16(37.3)$ & 0.265 & $9(34.6)$ & $7(27)$ & \\
\hline Positive & $7(16.3)$ & $10(23.2)$ & & $5(19.2)$ & $5(19.2)$ & \\
\hline \multicolumn{7}{|l|}{$\mathrm{C}$, Metastatic variables, n (\%) } \\
\hline Metastatic site & & & & & & 0.304 \\
\hline Liver + bone & $8(21)$ & $4(10.5)$ & 0.032 & $4(19)$ & 0 & \\
\hline Liver + lung & $8(21)$ & $13(34.2)$ & & $7(33.4)$ & $6(28.6)$ & \\
\hline Liver + lung + bone & - & $3(7.9)$ & & - & $3(14.3)$ & \\
\hline Liver + lung + brain & $1(2.7)$ & $1(2.7)$ & & - & $1(4.7)$ & \\
\hline Metastatic tumor size $(\mathrm{cm})$ & & & & & & 0.295 \\
\hline $0-1.0$ & $4(9.3)$ & $8(18.6)$ & 0.341 & $6(23.1)$ & $2(7.7)$ & \\
\hline $1.1-3.0$ & $4(9.3)$ & $7(16.3)$ & & $3(11.4)$ & $4(15.4)$ & \\
\hline$>3.0$ & $9(20.9)$ & $11(25.6)$ & & $5(19.2)$ & $6(23.1)$ & \\
\hline Metastatic tumor number & & & & & & 0.121 \\
\hline 1 & $3(7)$ & $5(11.6)$ & 0.027 & $3(11.4)$ & $2(7.8)$ & \\
\hline $2-3$ & $5(11.6)$ & $4(9.4)$ & & $3(11.4)$ & $1(3.9)$ & \\
\hline$>3$ & $9(20.9)$ & $17(39.5)$ & & $8(30.9)$ & $9(34.6)$ & \\
\hline
\end{tabular}

All data are expressed as mean \pm SD (range) of the mean of individual groups and Mann-Whitney U test and Chi-square test were used. Pathologic stage was determined in accordance with the 7th edition of the International Tumor-Node-Metastasis (TNM) system. Histologic

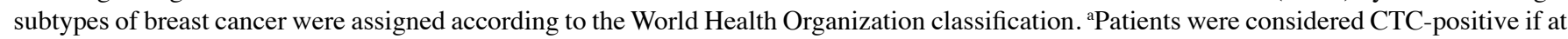
least five CTCs/7.5 ml were found. 'Pooled correlation between negative and positive CTC groups were analyzed by Spearman's rank correlation coefficient. 'Pooled correlation between low- and high-CTC groups were analyzed by Spearman's rank correlation coefficient. BCLM, breast cancer liver metastasis; CTCs, circulating tumor cells; BMI, body mass index; PET/CT, positron emission tomography/computed tomography; SUVMax, maximum standardized uptake value; ER, estrogen receptor; PR, progesterone receptor; HER2, human epidermal growth factor receptor 2 . 
43 patients) of patients were categorized as positive-CTCs ( $\geq 5$ CTCs $/ 7.5 \mathrm{ml}$ blood) and $40 \%$ (17 of 43 patients) of patients were categorized as negative-CTCs $(<5$ CTCs $/ 7.5 \mathrm{ml}$ blood). Meanwhile, almost $46 \%$ (12 of 26 patients) of patients were categorized as high-CTCs ( $>15 \mathrm{CTCs} / 7.5 \mathrm{ml}$ blood) and $54 \%$ (14 of 26 patients) of patients were categorized as low-CTCs ( $\geq 15 \mathrm{CTCs} / 7.5 \mathrm{ml}$ blood). Comparison of patients with positive/negative CTCs and those with high/low CTCs showed similar age, body mass index (BMI), disease duration, and SUVmax of PET/CT scan. However, there were more patients with low-CTCs than those with high-CTCs in both the newly diagnosed and the recurrent metastasis groups $(\mathrm{P}=0.04)$. Histologically, more than $65 \%$ of all samples were hormone receptor positive, either ER- or PR-positive (30 of 43 patients), and HER2-negative (26 of 43 patients). Metastases to the bone, lung, and brain were sufficiently frequent for statistical analysis. Sites of involvement in the 43 patients were: Liver + bone (12 patients), liver + lung ( 21 patients), liver + lung + bone ( 3 patients), and liver + lung + brain (2 patients). The calculated detection efficiency of CTCs was $>61 \%$ when 6-24 tumor cells were present per $7.5 \mathrm{ml}$ peripheral blood (26 of 43 patients), and there was a $100 \%$ success rate in the detection of histopathological variables from captured tumor cells. In this study, the median CTCs was 32.35 per $7.5 \mathrm{ml}$ (interquartile range $0-248$ ). Interestingly, newly diagnosed metastatic patients had a higher number of CTCs than recurrent metastatic patients (Fig. 2A, mean CTC count 38.30 vs. $25.50 ; \mathrm{P}=0.032$ ).

We performed a linear logistic regression analysis to predict the accuracy of CTC detection and characteristic of BCLM. Fig. 2B shows that the average recovery was calculated to be $81 \%\left(R^{2}=0.91\right)$. We assessed possible sources of cell loss and found that nearly $10 \%$ of cells were in the waste from cell isolation and sampling, and another 9\% of cells died in the magnetic tubing. Therefore, counting of CTCs may not identify patients with a high risk of postoperative recurrence but could be a more precise indication for the diagnosis for BCLM.

Clinicopathological confirmation of newly diagnosed and recurrent liver metastases. To confirm liver metastases of newly diagnosed and recurrent MBC, ${ }^{18} \mathrm{~F}$-FDG PET/CT scan was conducted. Serial biopsy sections from paraffin blocks of different regions of resected or punctured tumor samples were examined by H\&E staining. Pathologically, all breast cancer samples were identified as invasive ducal carcinoma samples. Of the 43 patients, 23 newly diagnosed and 20 postoperative recurrent liver metastases were found by ${ }^{18} \mathrm{~F}-\mathrm{FDG}$ PET/CT. The SUVmax of PET/CT between the the negative-CTC group was not significantly different than that of the positive-CTC group (6.12 \pm 3.44 vs. $5.97 \pm 2.82 ; \mathrm{P}=0.39$ ). Similarly, no significant difference was seen in the SUVmax between the low-CTC and high-CTC groups ( $4.59 \pm 3.27$ vs. $4.96 \pm 2.73 ; \mathrm{P}=0.76$ ) (Table I). Representative H\&E staining and ${ }^{18} \mathrm{~F}-\mathrm{FDG}$ PET/CT images of typical newly diagnosed MBC patient no. 13 (BCLM13H, Table SI) showed this patient to be in the high-CTC group while recurrent MBC patient no. 3 (BCLM03L, Table SI) belonged to the low-CTC group. There was high proliferation of bile duct cells and infiltrating inflammatory cells in newly diagnosed liver metastases (Fig. 3A). Large necrotic, dense lymphocytic infiltrate and desmoplastic rim are the main structural depolarization in recurrent liver metastases (Fig. 3B). A significant increase in the number of hepatocytes and significant diffuse infiltration by poorly differentiated carcinoma cells were evident in connective tissues from both newly diagnosed and recurrent liver metastatic groups. The ${ }^{18}$ F-FDG PET/CT images clearly show that newly diagnosed liver metastases (Fig. 3C) and the recurrent liver metastases (Fig. 3D) have similar imaging characteristics and SUVmax value. A meaningful increase in necrotic area in metastatic hepatic lesions on the right lobe of the liver was a prominent feature in recurrent liver metastases.

CTC isolation and morphological characterization. The $7.5 \mathrm{ml}$ blood samples from BCLM patients were used for CTC isolation using high efficiency Cell Search technology. We evaluated the quality of the blood samples before CTC isolation and none of the samples showed hemolysis. The stored CTC suspension was placed under a fluorescence microscope to select individual CTCs. Physical and three-color immunofluorescent characterization of CTCs isolated from patients with BCLM are shown in Fig. 4. There was a large number of cell debris under fluorescence microscope $(>75 \%)$ and few cells with intact morphology $(>11 \%)$. The basis for affinity-binding systems used for CTC enrichment and identification is the selection of specific tissue-type and cancer-specific markers, such as leukocyte (WBC)-expressed cell membrane CD45 (a tyrosine phosphatase) and epithelial cell (EPC)-expressed cytoplasmic CK-8, as well as DAPI nuclear staining. Then, we further distinguished the EPCs from CTCs by size, where the cutoff was a cellular diameter $<8 \mu \mathrm{m}$ for CTCs and a diameter $>8 \mu \mathrm{m}$ for EPCs $(27,39,40)$. Morphologically, CTCs from BCLM patients were recognizable with typical deformations of a neoplastic cell: Hyperchromatic nuclei by fluorescence microscopy with an irregular shape, high nuclear-to-cytoplasmic ratio, as described by Krebs et al (41). Enriched CTCs can be isolated based on their distinct physical (size or deformability) or fluorescence properties from EPCs and WBCs (Fig. 4). The CTC sample appears as $\mathrm{CD} 45^{-} / \mathrm{Nucleus}^{+} / \mathrm{CK}^{+}$, while WBCs appear as $\mathrm{CD}^{+} 5^{+} / \mathrm{Nucleus}^{+} / \mathrm{CK} 8$. Cells that stain $\mathrm{CD}^{\circ} / \mathrm{Nucleus}^{+} / \mathrm{CK}^{+}{ }^{+}$with larger diameter $<8 \mu \mathrm{m}$ were identified as EPCs. Furthermore, the dead cell mass sample shows cytoplasmic $\mathrm{CK}^{+}$. Obviously, the triple-immunostaining method is slightly limited by the specificity and availability of antibodies. Of a total of 2,081 captured cells from all samples, $1,359(65.3 \%)$ of the tumor cells were identified as CTCs, 430 (20.7\%) as WBCs, and $292(14.1 \%)$ as EPCs. The details of the CTC count from each BCLM patient are shown in the Table S1.

Correlation of CTCs and clinicopathological characteristics. By comparing the negative- and positive-CTC groups, the initial disease stage and HER2 status were strongly correlated with the presence of CTCs $(R=0.75, P=0.04$ and $R=0.72$, $\mathrm{P}=0.032$, respectively). These findings suggest that the CTC count may be a prognostic factor for survival of BCLM patients. Strikingly, by comparing the low- and high-CTC groups, it was found that the CTC number was significantly correlated with the size of metastasis and metastatic tumor number $(\mathrm{R}=0.81, \mathrm{P}=0.03$ and $\mathrm{R}=0.77, \mathrm{P}=0.03$, respectively). These data indicate that CTC counting could be a viable 
A

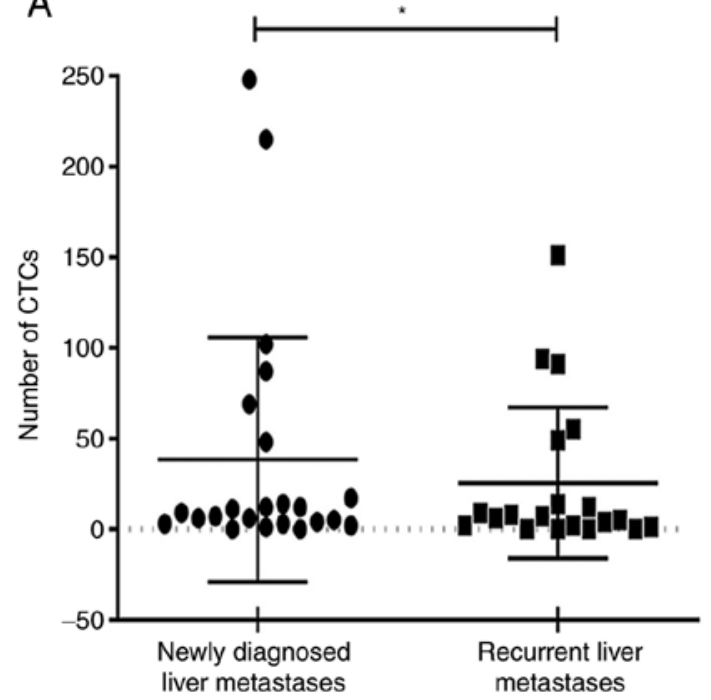

B

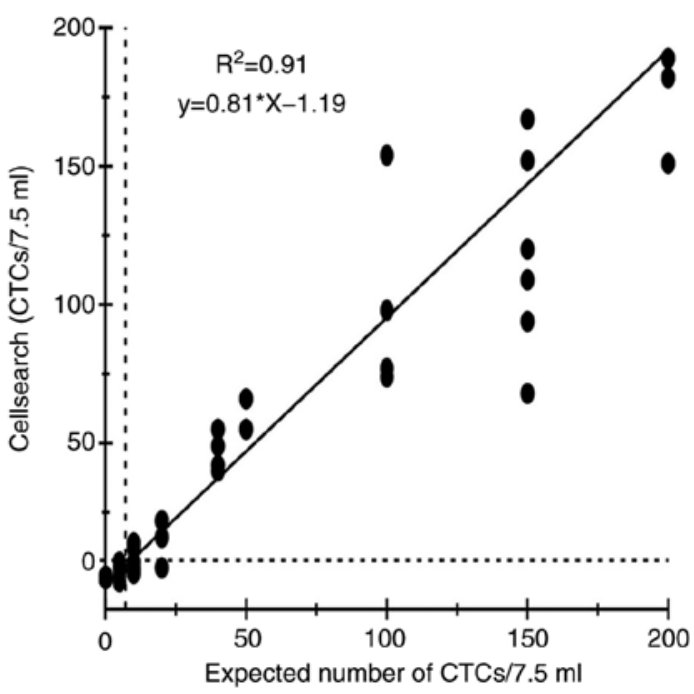

Figure 2. Analysis of CTCs in different patient samples. (A) Comparison of the CTC count between newly diagnosed liver metastases (n=23) and recurrent liver metastases $(n=20)$ in a $7.5 \mathrm{ml}$ blood sample of BCLM patients $(n=43)$. In general, higher percentages of CTCs were observed in newly diagnosed BCLM patients (mean CTCs in newly diagnosed metastases 38.30 vs. 25.50 in recurrent metastases). (B) Linear logistic regression of CTC detection. Average (black line) CTC recovery was calculated by using the linear logistic regression from 43 BCLM patients. The cut-off levels (10 CTCs for expected and 5 CTCs for CellSearch per sample) are indicated by the dashed lines. ${ }^{*} \mathrm{P}<0.05$. CTCs, circulating tumor cells; BCLM, breast cancer liver metastasis.

A

Newly diagnosed liver metastases

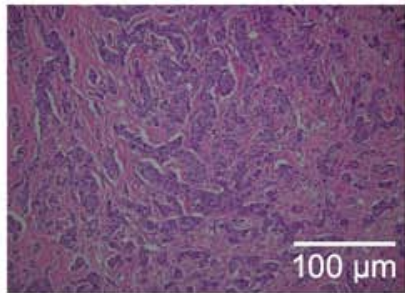

C

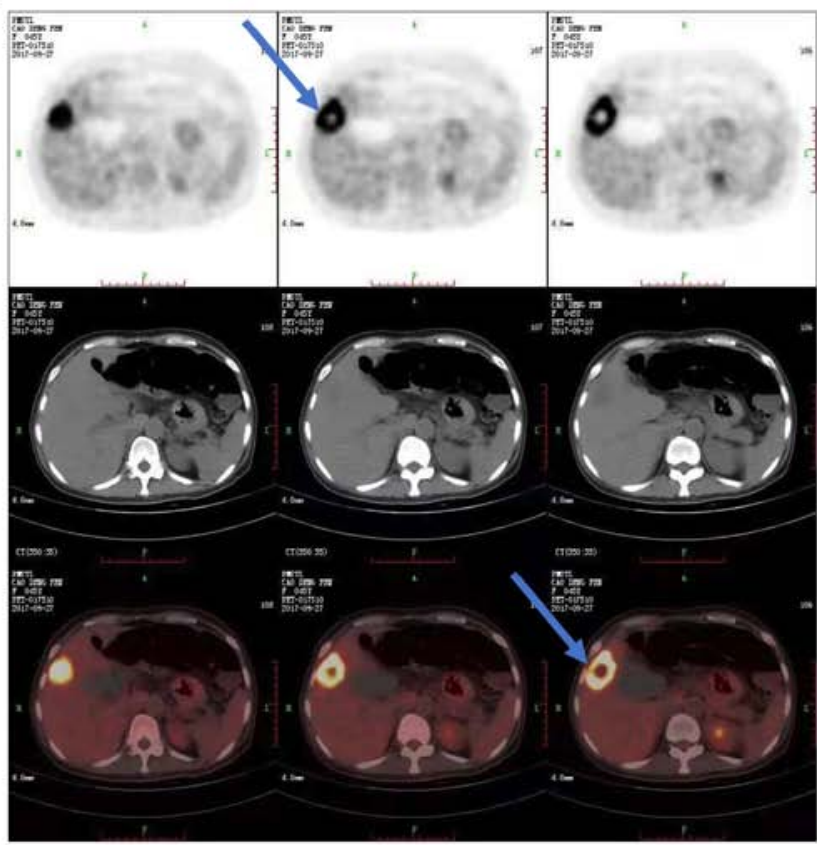

B
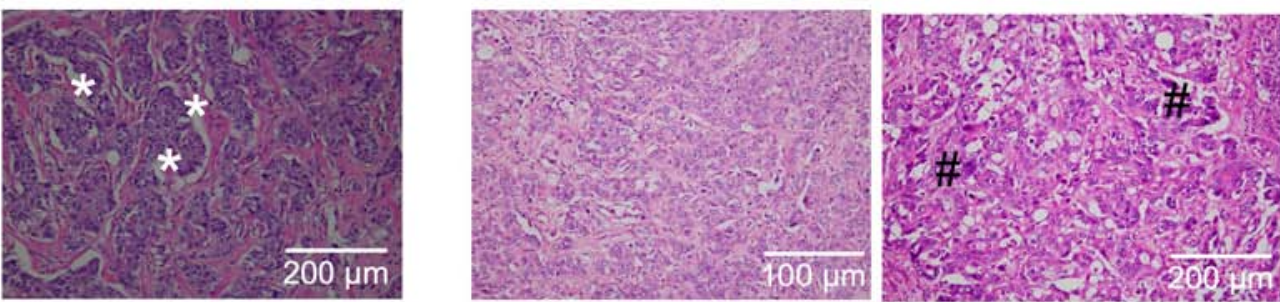

D

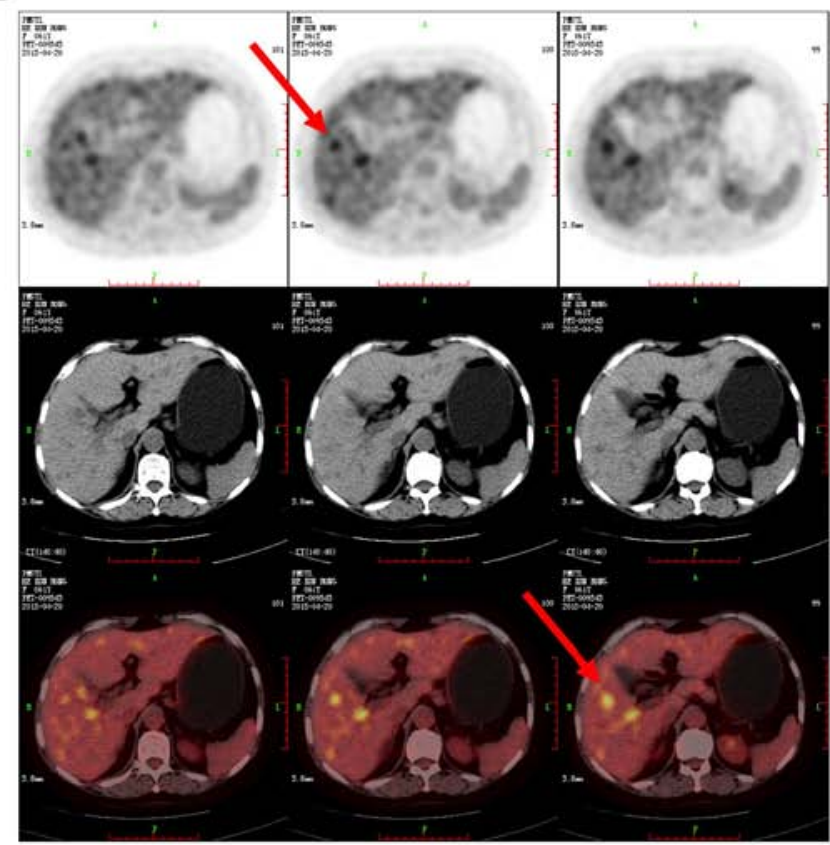

Figure 3. Representative images of histological staining and 18F-FDG PET/CT imaging of a newly diagnosed liver metastases (left panel: A and C) and recurrent liver metastases (right panel: B and D). (A and B) H\&E staining showed the presence of a large fibrotic focus (*) in the primary tumor and of dense lymphocytic infiltrate and desmoplastic rim (\#) in liver metastasis cells. (C and D) The 18F-FDG PET/CT images clearly show the preoperative primary tumor in the right breast (blue arrows) and the metastatic post-treatment tumor in the right lobe of the liver (red arrows). 18F-FDG PET/CT, fluorine-18-fluorodeoxyglucose positron emission tomography/computed tomography; $\mathrm{H} \& \mathrm{E}$, hematoxylin and eosin. 

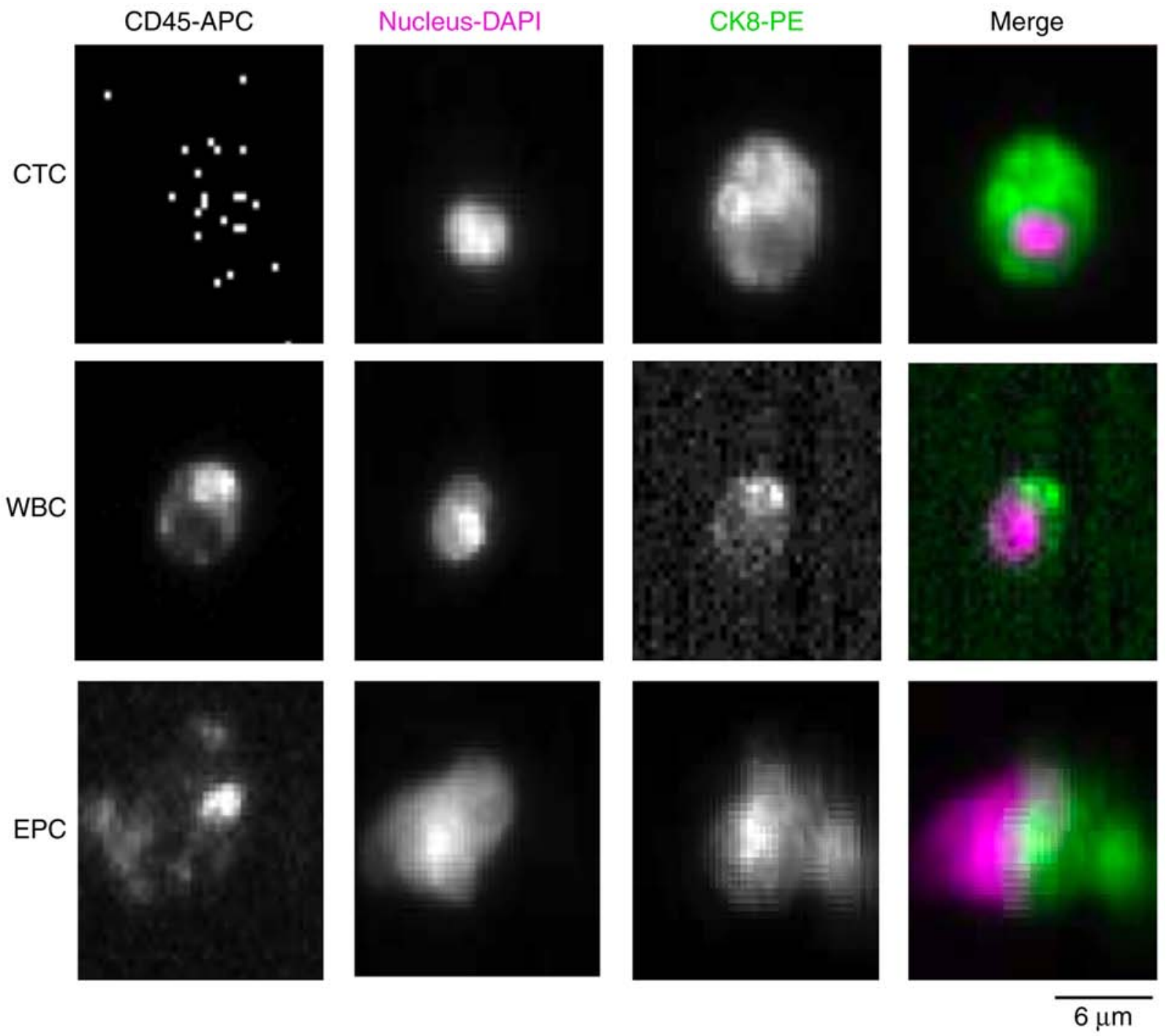

Figure 4. Gallery of fluorescent immunostaining of CTCs, EPCs and leukocytes (WBCs) from patients with BCLM. Cells were stained by antiCD45-APC antibody, 4',6-diamidino-2-phenylindole (DAPI, nuclear staining; pink color), anti-CK8-PE (green color). The CTC sample is characterized by a $\mathrm{CD} 45 / \mathrm{Nucleus}^{+} / \mathrm{CK}^{+}$of a diameter $<8 \mu \mathrm{m}$, while $\mathrm{CD}^{-} 5^{+} / \mathrm{Nucleus}^{+} / \mathrm{CK} 8$ are identified as WBCs. Cells that stain $\mathrm{CD} 45 / \mathrm{Nucleus}^{+} / \mathrm{CK}^{+}{ }^{+}$are identified as $\mathrm{EPCs}$ with a diameter $<8 \mu \mathrm{m}$ (magnification $200 \mu \mathrm{m}$ ). BCLM, breast cancer liver metastasis; CTCs, circulating tumor cells; CK, cytokeratin; EPCs, epithelial cells; WBCs, white blood cells.

method by which to monitor distant metastasis. Additionally, we did not find any significant correlation between CTC presence or number and hormone receptor in the multivariate analysis model (Table II). In general, high CTC frequencies were correlated with disease severity and metastatic progression, which also suggests an effective value for CTCs in predicting the prognosis of BCLM.

Genome-wide CNVs of newly diagnosed and recurrent BCLM. Visualization of 10x genome-wide gene copy numbers was performed in CTC specimens of the newly diagnosed MBC patient no. 13 (no. BCLM13H, Table S1) and CNVs of recurrent MBC patient no 3 (no. BCLM03L, Table S1). We then applied 30x WGS sequencing of liver metastases from these two patients. Our primary finding showed that the average 'unique mapped of raw' of the single cell genome sequencing products was $79.58 \%$ (78.74-80.17\%), which further implies that uniform amplification of a single cell genome can be used for genomic copy number analysis. As shown in Fig. 5, the
CNV pattern of CTCs and liver metastases in newly diagnosed and recurrent $\mathrm{MBC}$ patients were significantly different. A few $\mathrm{CNVs}$ were found in either the newly diagnosed liver metastases (Fig. 5A) or the recurrent liver metastases (Fig. 5C), in which gain and loss regions accounted for $\sim 7.4$ and $\sim 0.26 \%$ of their entire genomes, respectively. Remarkably, the region of 0-22,800 kbp of chromosome 13 and 0-22,750 kbp of chromosome 14 were the most significant CNVs in newly diagnosed and recurrent liver metastases, respectively. In comparison with CTCs of recurrent BCLM, there are nearly $\sim 45 \%$ gain and $\sim 4.7 \%$ loss regions in the CTCs isolated from the newly diagnosed metastases group (Fig. 5B and D).

In the CTCs of recurrent BCLM, the copy number slightly increases in most CNV regions with 1 copy as the lower limit and 3 copies as the upper limit (Fig. 5D). In addition, we also found a few regions with copy number between 3-5 copies located on chromosomes 9,8,11,13,14,15, and 22 (Fig. 5D). Other regions with copy numbers between 5-9 on these chromosomes accounted for $\sim 2.4$ and $\sim 2.1 \%$ of the 
Table II. Correlation between CTC count and clinicopathological features of the BCLM patients.

\begin{tabular}{lcccr}
\hline & \multicolumn{2}{c}{ CTC-negative and -positive } & & Low and high CTCs \\
\cline { 2 - 3 } Variable & $\mathrm{R}$ & P-value & $\mathrm{R}$ & P-value \\
\hline Subjects, $\mathrm{n}(\%)$ & 0.70 & 0.507 & 0.31 & 0.421 \\
Age (years) & 0.34 & 0.491 & 0.45 & 0.278 \\
BMI (kg/m ${ }^{2}$ ) & 0.28 & 0.204 & 0.67 & 0.602 \\
Disease durations (years) & 0.34 & 0.562 & 0.32 & 0.487 \\
PET/CT (SUVmax) & 0.28 & 0.762 & 0.44 & 0.395 \\
Initial disease stage & 0.75 & 0.041 & 0.72 & 0.382 \\
ER status & 0.66 & 0.582 & 0.40 & 0.071 \\
PR status & 0.28 & 0.231 & 0.37 & 0.542 \\
HER2 status & 0.72 & 0.032 & 0.64 & 0.265 \\
Metastatic site & 0.75 & 0.304 & 0.62 & 0.341 \\
Metastatic tumor size (cm) & 0.59 & 0.295 & 0.81 & 0.032 \\
Metastatic tumor number & 0.39 & 0.121 & 0.77 & 0.027 \\
\hline
\end{tabular}

Pooled correlation between negative/positive and low/high CTC groups were analyzed by Spearman's rank correlation coefficient. BCLM, breast cancer liver metastasis; CTC, circulating tumor cell; BMI, body mass index; PET/CT, positron emission tomography/computed tomography; SUVMax, maximum standardized uptake value; ER, estrogen receptor; PR, progesterone receptor; HER2, human epidermal growth factor receptor 2 .

A Newly diagnosed liver metastases

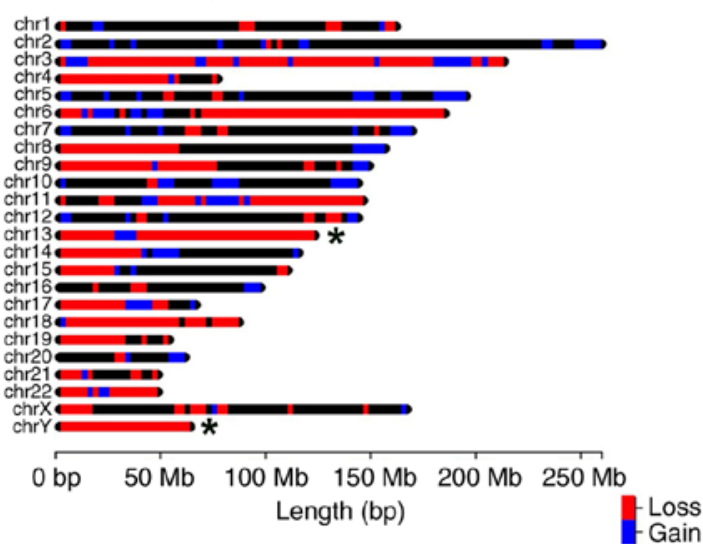

C

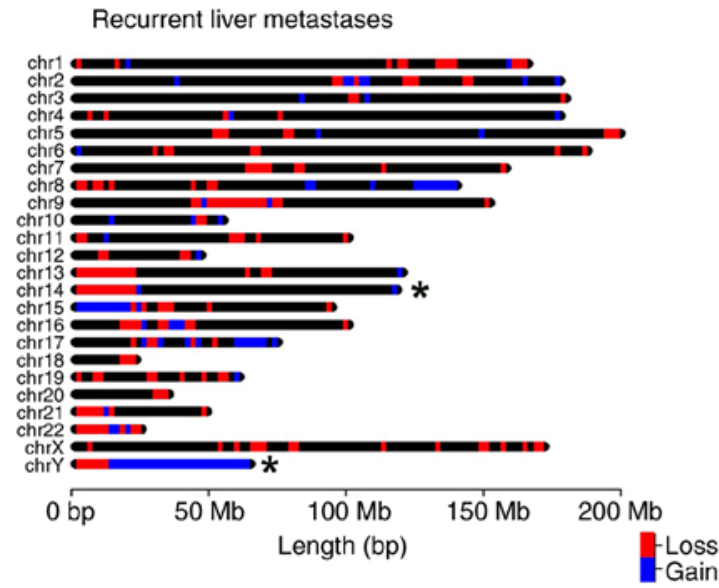

B

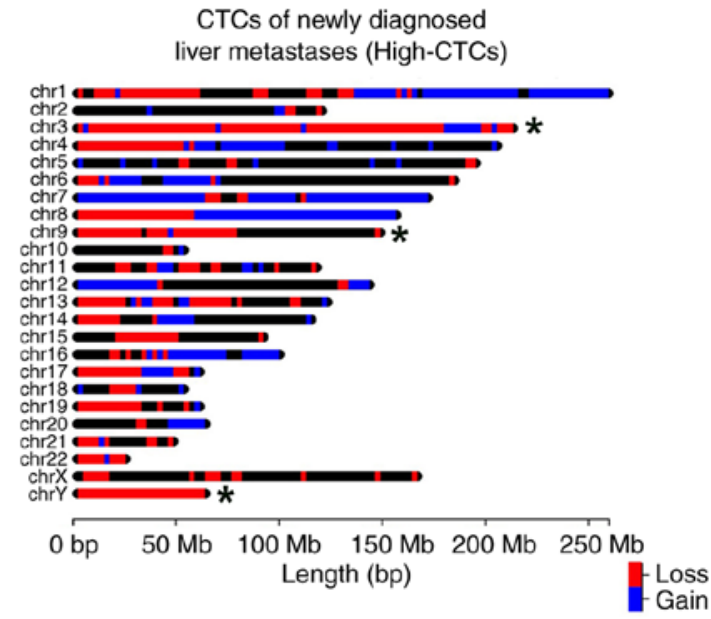

$\mathrm{D}$

CTCs of recurrent

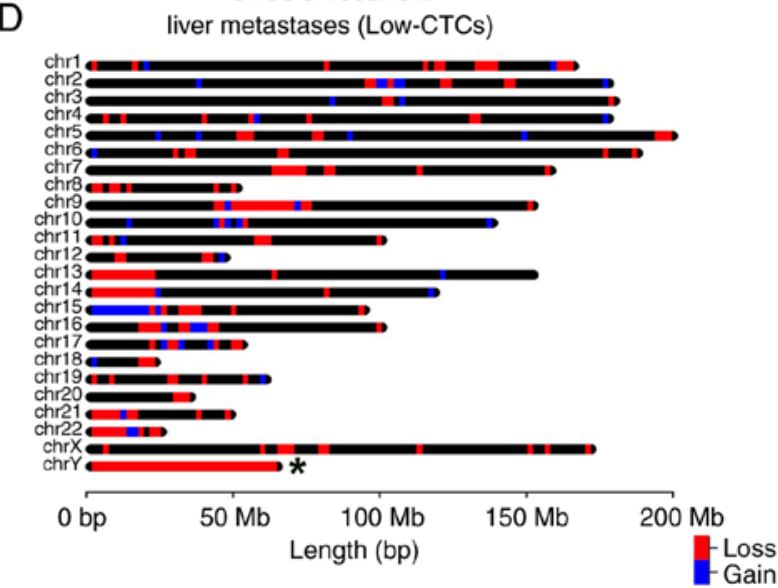

Figure 5. Visualization of 10x genome-wide gene copy number analysis between newly diagnosed liver metastases (A) CTCs from newly diagnosed liver metastases (B) recurrent liver metastases (C) and CTCs from recurrent liver metastases (D) The histogram shows the frequency of genomic gains (blue) and losses (red) of CTCs. Most significant CNVs in each group are shown with star symbol $(*)$. CTCs, circulating tumor cells; CNVs, copy number variations. 
A

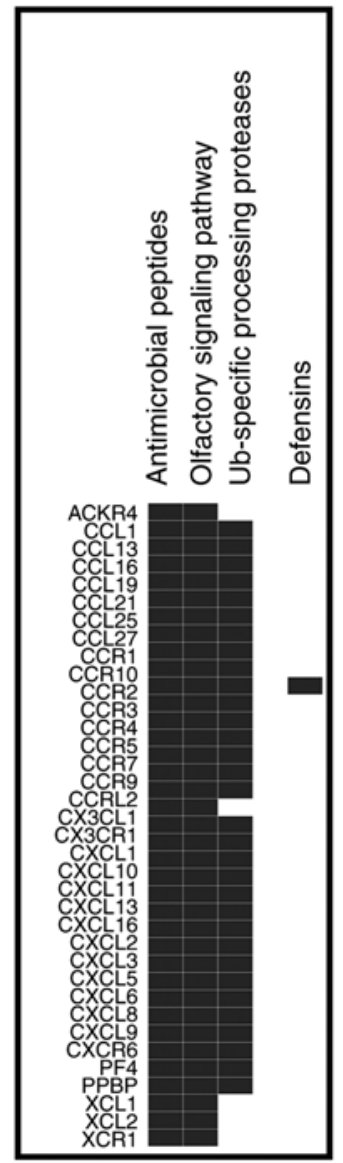

Newly diagnosed liver metastases
B

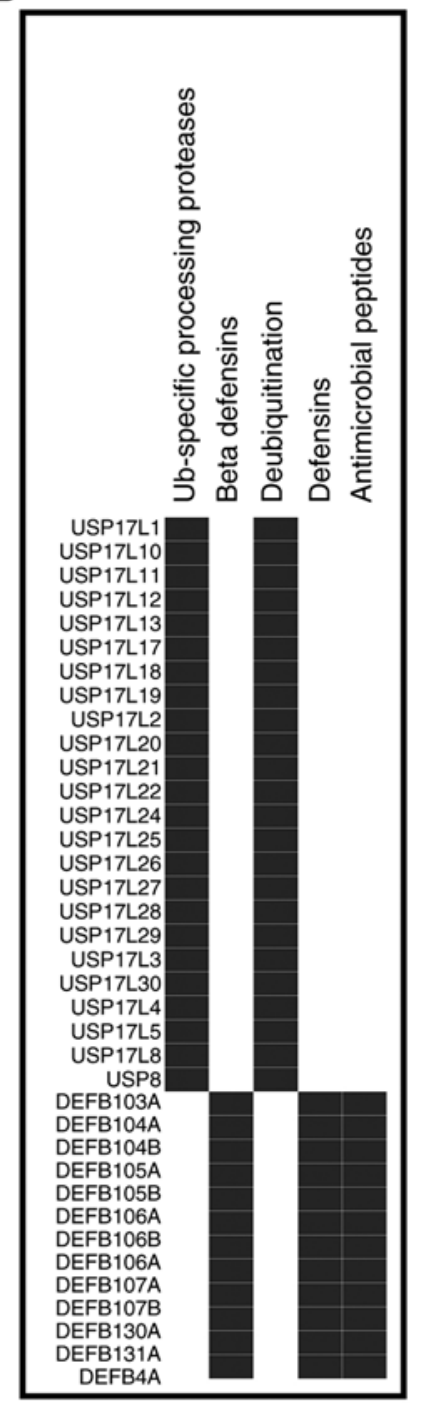

CTCs of newly diagnosed liver metastases
C

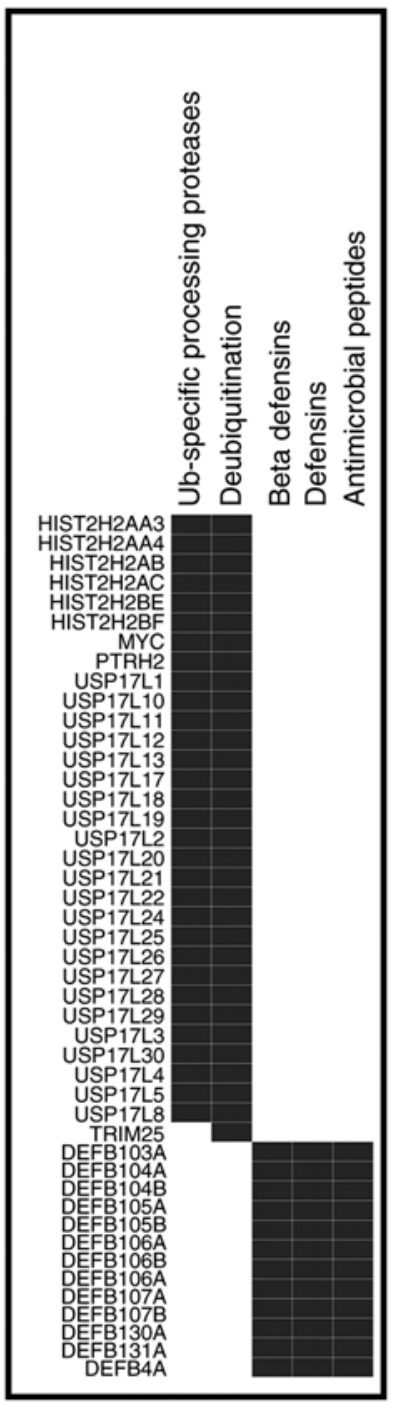

Recurrent liver metastases
D

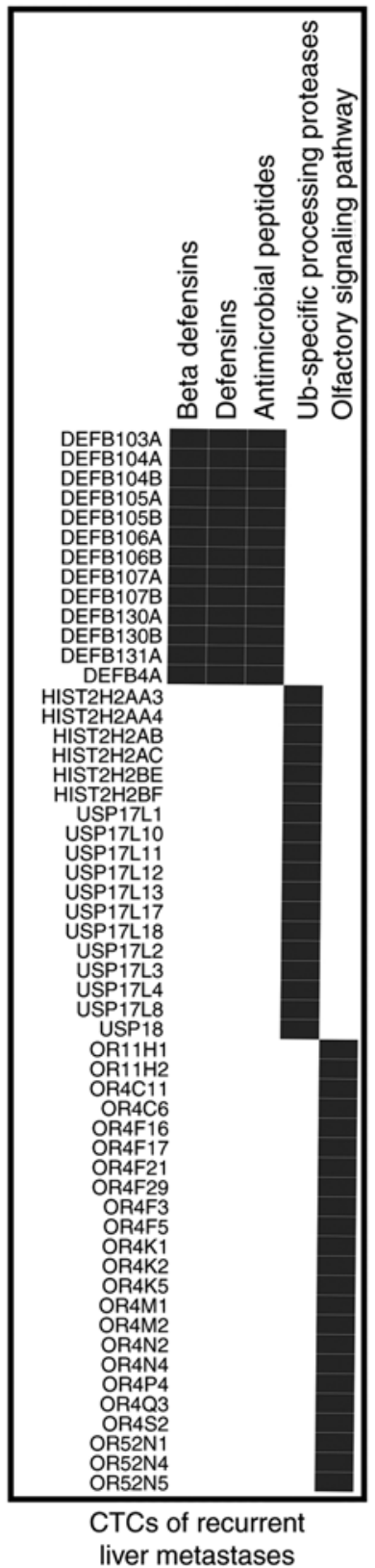

Figure 6. Heat map representation of CTC-shared CNVs in newly diagnosed liver metastases (A) CTCs of newly diagnosed liver metastases (B) recurrent liver metastases (C) and CTCs of recurrent liver metastases (D) Heat map representation of frequently deregulated genes in different groups. CTCs, circulating tumor cells; $\mathrm{CNVs}$, copy number variations.

entire genome (Fig. 5C). Nearly all CNVs that arise in newly diagnosed liver metastases (Fig. 5A) were found in CTCs of newly diagnosed BCLM (Fig. 5B), but there are many more CNVs in newly diagnosed liver metastases. Furthermore, in CTCs of newly diagnosed liver metastases, chromosomes 3 , 9 and $\mathrm{Y}$ had significant CNVs (Fig. 5B). In contrast, the CNV pattern of isolated CTCs of recurrent BCLM patient (Fig. 5C) was similar to recurrent liver metastases (Fig. 5D) (nearly 82\% of the gain/loss regions). The CNVs on the remaining chromosomes showed some similarity between CTCs of recurrent BCLM and recurrent liver metastases.

Functional enrichment analysis of CNVs in newly diagnosed and recurrent BCLM. GSEA analysis was performed for a functional assessment of the genes involved in specific CTC-shared CNVs. The significant biological pathways are sorted in Table III and Fig. 6 . We found four common target pathways: $\beta$-defensins (hBDs), defensins, antimicrobial peptides, and Ub-specific processing proteases between newly diagnosed liver metastases (Fig. 6A), recurrent liver metastases (Fig. 6C), CTC-shared CNVs of newly diagnosed BCLM (Fig. 6B), and CTC-shared CNVs of recurrent BCLM (Fig. 6D). High expression of genes involved in defensin and hBD functions were enriched in all four groups. Moreover, we found chemokine receptors bind chemokines, GPCR ligand binding, $\mathrm{G}$ alpha (i) signaling events, and class $\mathrm{C} / 3$ (metabotropic glutamate/pheromone receptors) pathways with their response genes were higher in the CNVs of newly diagnosed 

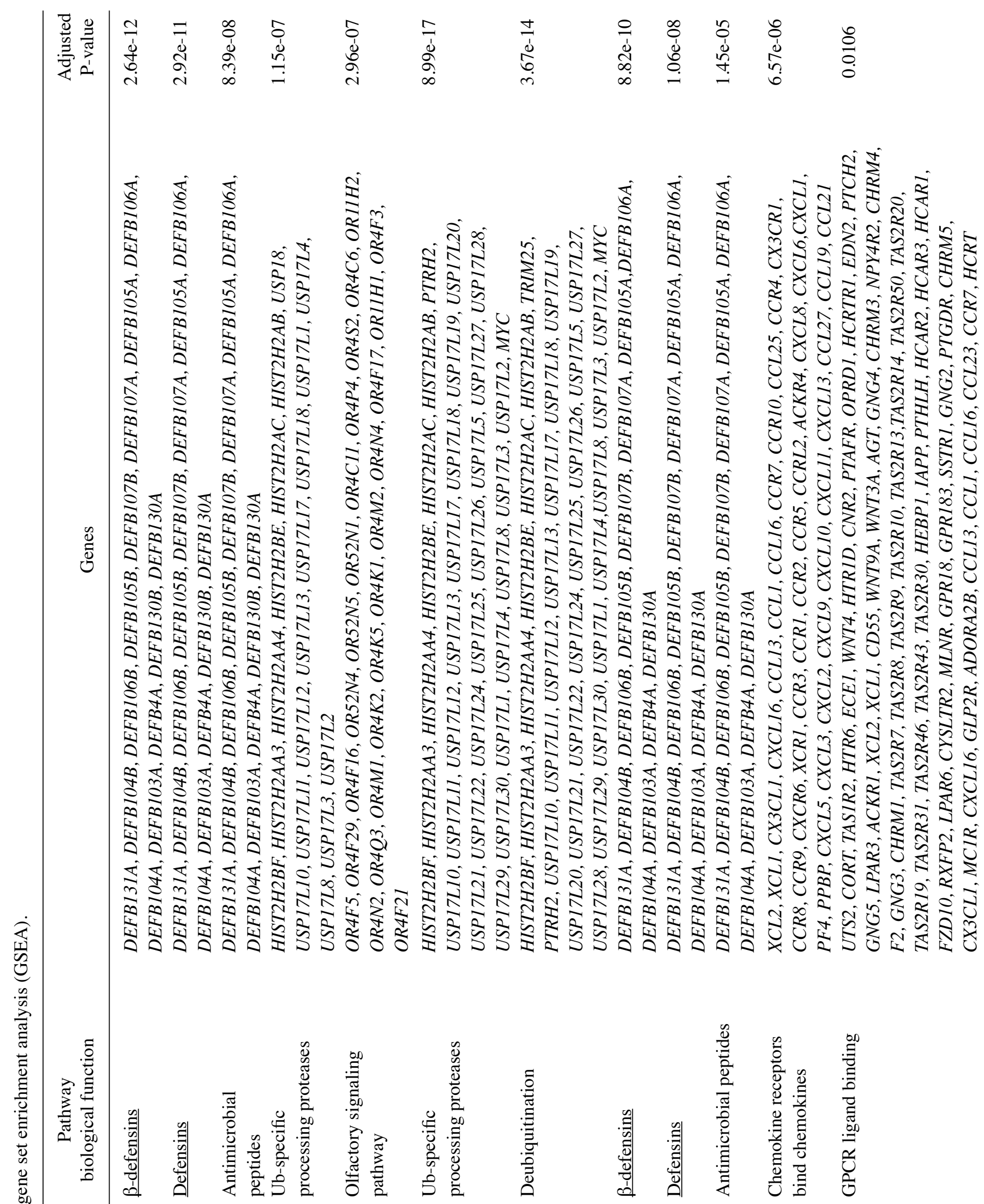

ఏี 

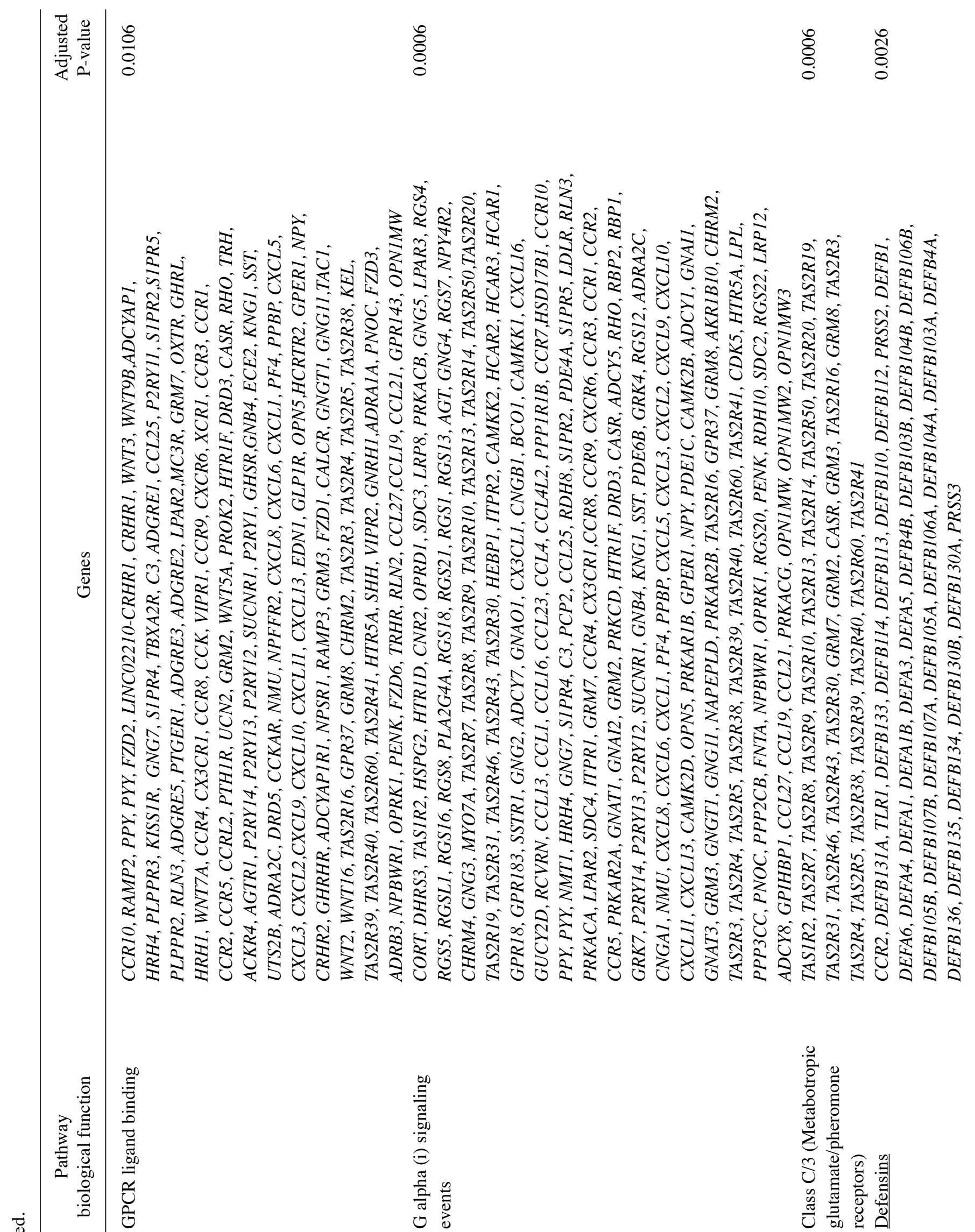


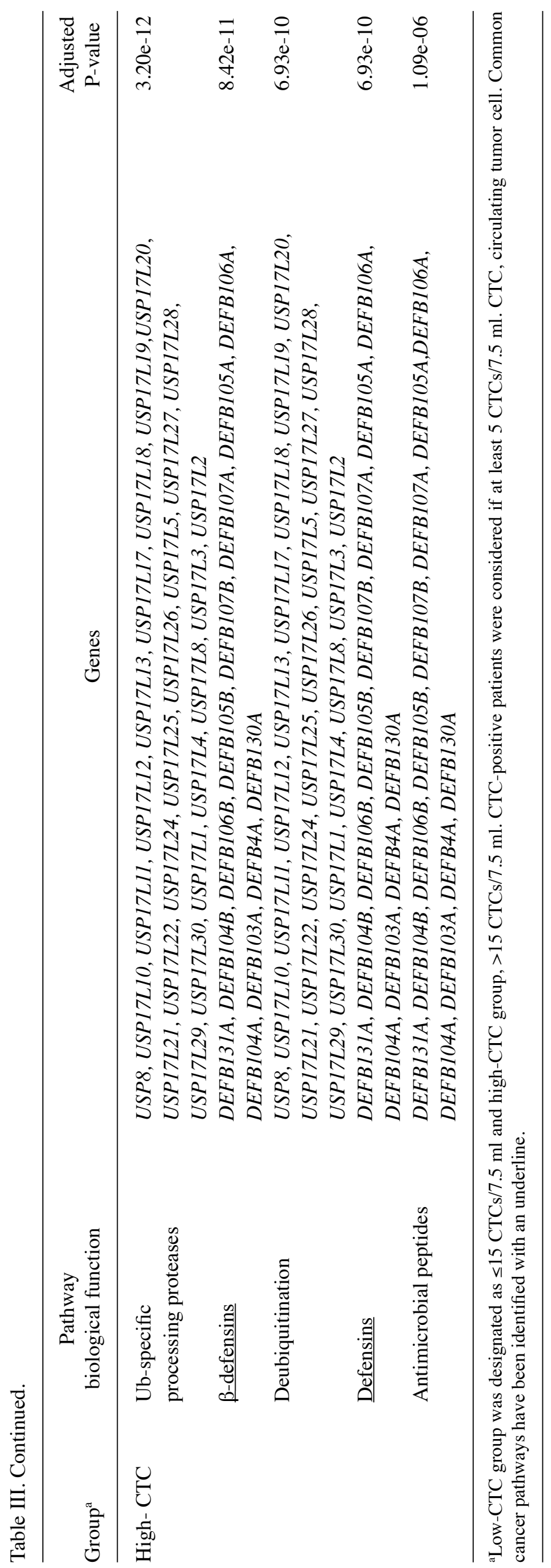


liver metastases compared with CNVs of recurrent liver metastases; which may partly account for the poor prognosis in newly diagnosed BCLM patients (Fig. 6).

Protein-protein interaction network analysis of CTC-shared $C N V s$. A pathway analysis and protein-protein interaction (PPI) networks of commonly dysregulated genes are presented in Figs. 7 and 8, receptively. Our findings show that GPCR ligand binding, chemokine receptor binds chemokine, and $\mathrm{G}$ alpha signaling events were enriched in patients with liver metastatic phenotype, both newly diagnosed and recurrent (Fig. 7A and C). Whereas Ub-specific processing protease, deubiquitinating, anti-microbial peptides, defensins and hBDs pathway/biological function were enriched in CTC-positive patients (Fig. 7B and D). The emapplot (Fig. 8) and cnetplot of PPI analysis (Fig. S1) indicate that all these enriched pathways and genes may play similar and critical roles in the development and progression of liver metastasis. In addition, the enriched genes have previously been reported to play critical roles in anti-angiogenesis, immunomodulation, and cell growth. These findings show that these genes are involved in: i) antitumor immunity with non-immunogenic tumor antigens (anti-microbial peptide pathway enriched in the KEGG pathway analysis); ii) suppression of cell growth, cell migration via cell cycle arrest in G1/S checkpoint (hBDs and defensins are enriched in the KEGG pathway analysis); iii) intravasation competency through ECM remodeling and collagen catabolism (defensins and GPCR ligand binding are enriched in the KEGG pathway analysis).

\section{Discussion}

The present study, using a high-throughput whole genome sequencing (WGS) technique, provides the first comprehensive assessment of copy number variations (CNVs) among circulating tumor cells (CTCs) in patients with breast cancer liver metastasis (BCLM). In our analysis, which enrolled 43 breast cancer patients who had newly diagnosed or postoperative liver metastasis, we found that the presence of CTCs was associated with recurrence and a shorter disease-free survival time in patients with BCLM. The included cohort is the largest prospective study that counted CTCs to detect newly diagnosed and recurrent liver metastasis. Furthermore, we compared the consistency and efficiency of genome-wide $\mathrm{CNVs}$ with other common methods to distinguish newly diagnosed and recurrent BCLM. These findings highlight that higher CTC frequencies are correlated with disease severity and liver metastatic progression, thereby suggesting an effective value for CTCs in the detection of BCLM in comparison with common detection methods such as pathohistology and PET/CT imaging. Moreover, our data confirm that a higher number of CTCs isolated from BCLM patients is correlated with disease severity and metastatic progression, and is an independent prognostic factor associated with overall survival of BCLM patients. Analysis of biological pathways suggests that genes of defensins and hBDs are significantly associated with anti-angiogenesis, immunomodulation and antitumor cell growth signaling pathways; which are relevant in the development and progression of liver metastasis.
It is well established that CTC detection may be a valuable clinical biomarker in cancer-related processes such as angiogenesis, proliferation, differentiation, and metastasis $(25,42)$. Early detection of CTCs in cancerous serum has been introduced as a potential biomarker for the diagnosis of various types of cancer $(25,43,44)$. CTC detection in early stages of breast cancer can help identify patients with a high risk of recurrence after surgery and help the clinician to offer the best strategy for follow-up and treatment $(25,45)$. Recently, modern molecular biology detection technologies such as WGS and whole exome sequencing are among the most promising methods for the dissection of metastatic organotropism $(46,47)$. It has been widely accepted that profiling of CNVs with WGS is an accretive and reliable analytical tool that uses an ultra-low input of blood-derived DNA $(29,42,48,49)$. Likewise, CTC characterization by using genome-wide CNVs analysis provides more rapid and less expensive data collection, high diagnostic sensitivity and specificity, as well as advanced analysis of molecular phenomena, including fluorescence in situ hybridization for detection of tumor-specific genomic changes (47). The clinical value of CTC detection and enumeration in peripheral blood of cancer patients is an attractive and significant part of cancer biomarker research (50-52).

In the present study, the diagnostic value of genome-wide CNVs in BCLM was successfully confirmed with fluorescent-labeled antibodies that target tumor cell markers, and staining and washing were found to have little or no effect on the retention of tumor cells. In addition, we compared the consistency and efficiency of genome-wide CNVs with other conventional detection methods that distinguish between newly diagnosed and recurrent BCLM. This may provide a potentially valuable approach for the genetic characterization of newly diagnosed and recurrent BCLM. Additionally, CTC characterization by genome-wide CNVs allows tumor cells to be recovered for subsequent molecular analysis. Strikingly, our finding indicates that CTC counting could be a promising index for the monitoring of recurrent metastases. In general, higher CTC frequencies are significantly correlated with tumor severity and metastatic progression, which suggests the clinical value of CTCs for a variety of initial disease staging of BCLM patients.

We used the standard EpCAM method for isolating and distinguishing of the CTCs between primary breast cancer and newly diagnosed liver metastatic cancer. In the last decade, extensive resources and several methods have been invested into developing methods for detecting, enriching and characterizing of CTCs in different diseases (27,53-55). Methodologically, these techniques have many challenges that must be remedied, such as the need to improve purity, throughput, cell viability after recovery, and rates of enrichment. EpCAM-independent method is the first and most-available method for the detection of CTCs that has accelerated the development of numerous isolation technologies based on physical approaches and biological properties of CTCs $(56,57)$. On the other hand, non-EpCAM-based methods, such as dielectrophoresis, immunoaffinity-based methods and microfiltration are other isolation techniques with the advantage that they can enrich CTCs without EpCAM expression. Unfortunately, our current knowledge does not allow the possibility to identify, standardize and classify CTCs in cancer 
A

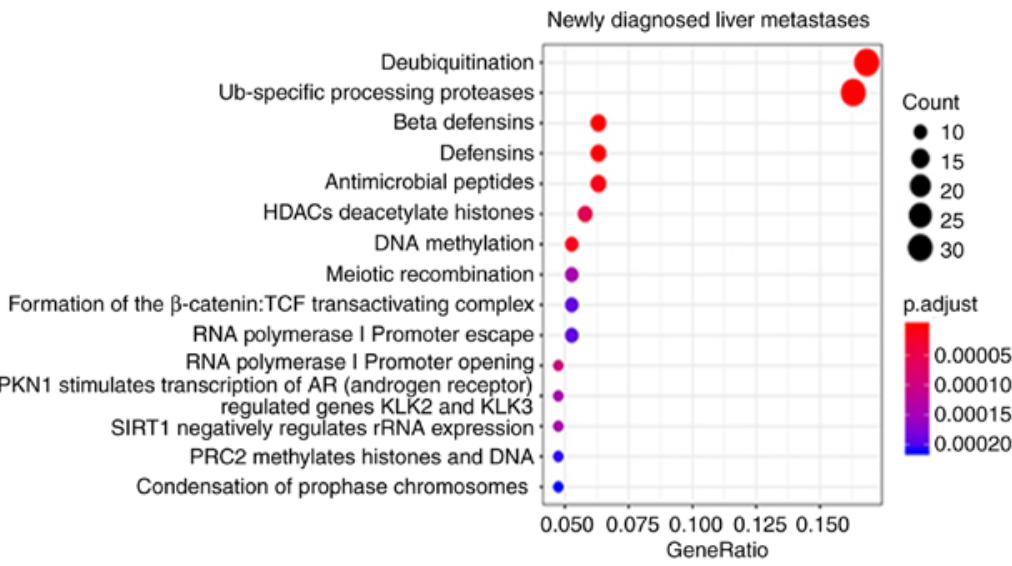

B

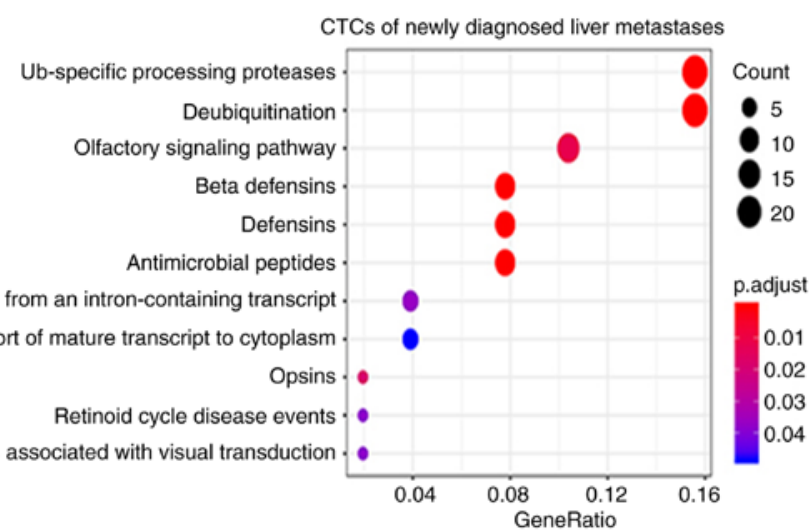

C
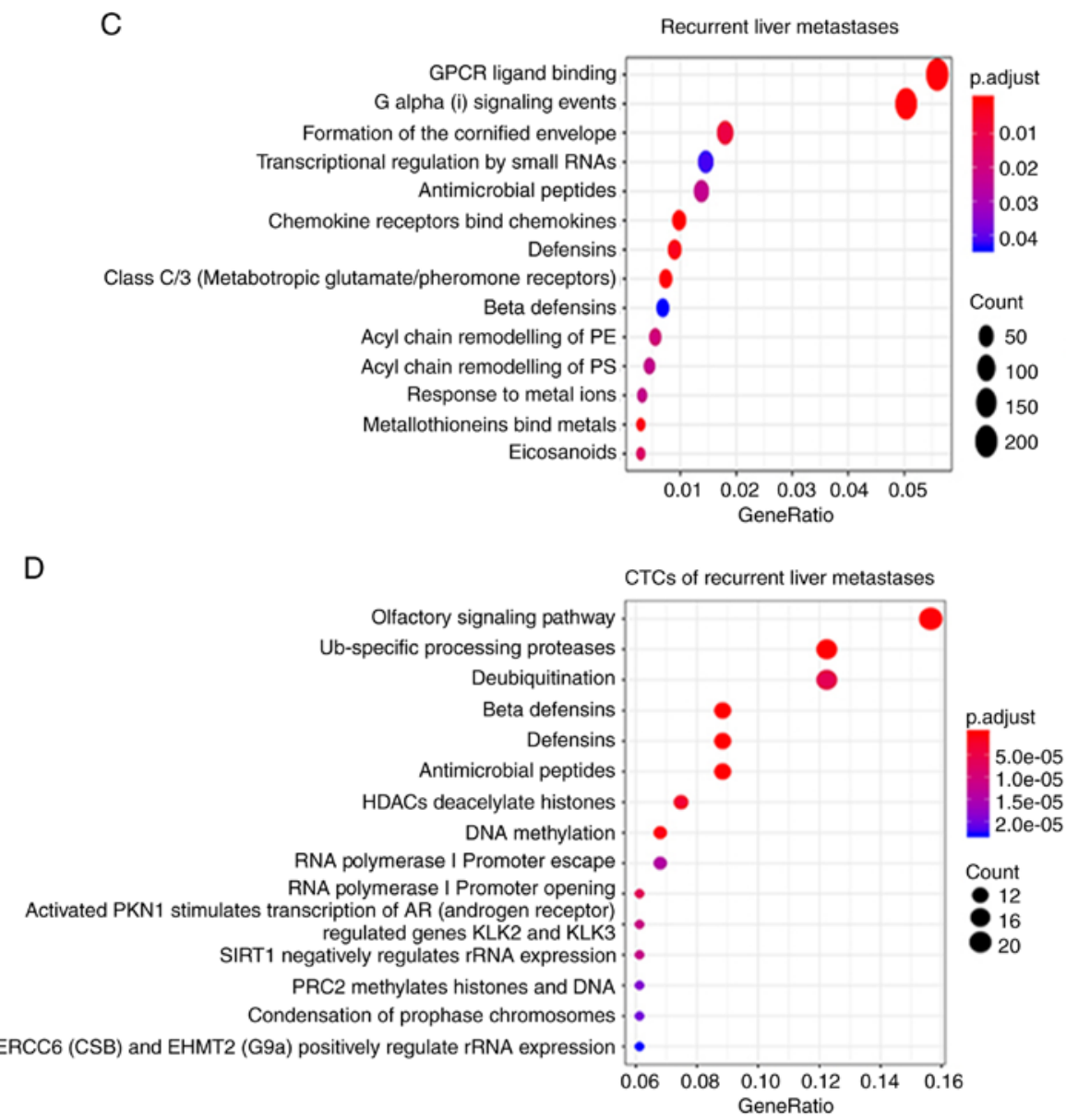

Figure 7. GSEA of different groups in newly diagnosed liver metastases (A) CTCs of newly diagnosed liver metastases (B) recurrent liver metastases (C) and CTCs of recurrent liver metastases (D) The size and color intensity of a circle represents the numbers of genes and -log 10 (P-value) for each group, respectively. GSEA, gene set enrichment analysis, CTCs, circulating tumor cells. 
A

\section{Newly diagnosed liver metastases}

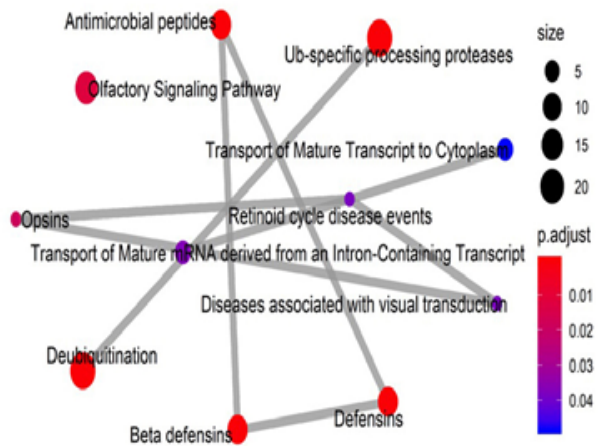

C

\section{Recurrent liver metastases}

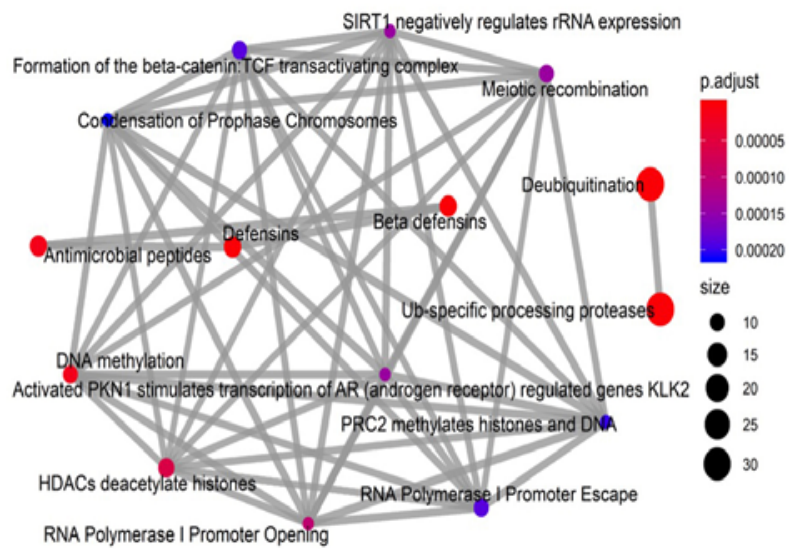

B

\section{CTCs of newly diagnosed liver metastases}

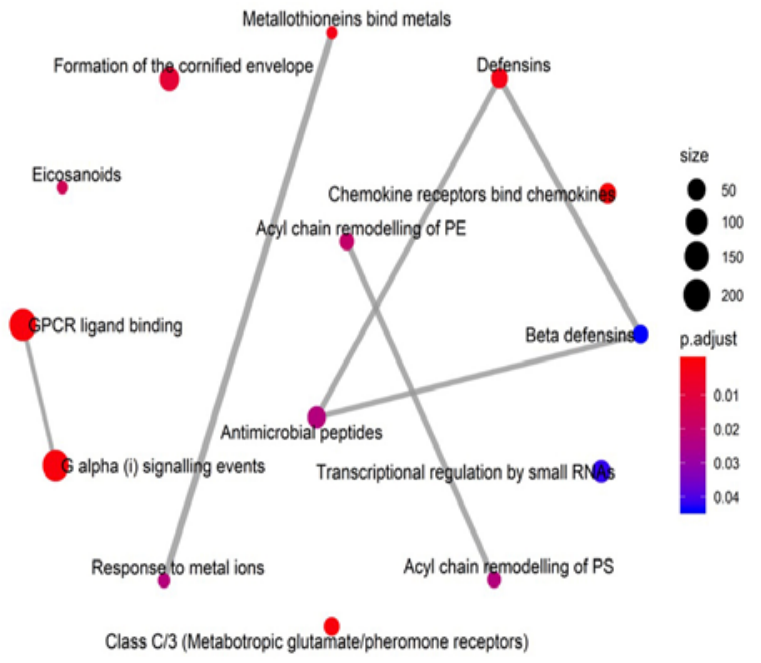

D

\section{CTCs of recurrent liver metastases}

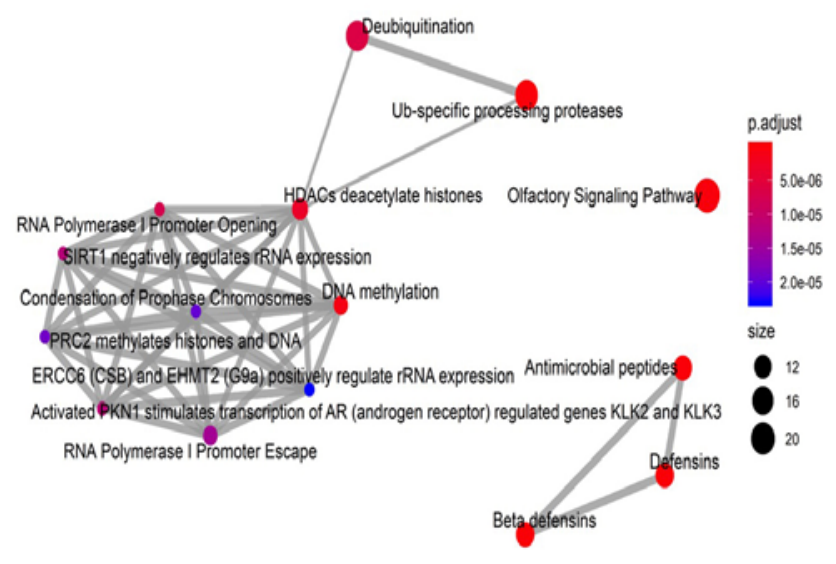

Figure 8. PPI network of commonly deregulated targeted genes and pathways in newly diagnosed liver metastases (A), CTCs of newly diagnosed liver metastases (B), recurrent liver metastases (C) and CTCs of recurrent liver metastases (D). Filled color represents the -log2 (fold change) of each gene, and border orange color represents the module to which each gene belongs. CTCs, circulating tumor cells; PPI, protein-protein interaction.

cells at different stages (58-60). Likewise, it would be desirable if these non-EpCAM-based methods or markers were able to distinguish between metastatic and non-metastatic CTCs. However, further improvements in pre-enrichment steps will develop methods for the capture and characterization of these cells. Surely, this information is most vital for clinical use, determining the prognosis of disease, making treatment decisions and evaluating the efficacy of BCLM therapy.

In this prospective study, we found that the CNV patterns across the genomes of recurrent liver metastases and their CTCs were highly consistent, with nearly $82 \%$ of the gain/loss regions shared among them, for an average of $72-16 \%$ of the whole genome. The homology finding shows that $\beta$-defensins (hBDs) were highly conserved and were predicted to be a globular domain that is involved in anti-angiogenesis, immunomodulation, and antitumor cell growth (61). In the CTC-shared CNVs of newly diagnosed liver metastases and newly diagnosed liver metastases, the CNVs were scattered across all chromosomes, but most of them were located on chromosome 13 and 14 (62). Furthermore, functional enrichment analysis of CTC-shared CNVs revealed that cell growth and cell migration via cell cycle arrest were closely related to recurrent and newly diagnosed metastases; which showed a similar phenotype with KEGG analysis. Importantly, high expression of genes that are involved in defensins and hBDs were enriched in all four groups. Identifying such adaptations has increased our knowledge of the role of hBDs in the process of metastasis of breast cancer $(61,63)$. Recently, studies have shown a potential role for hBDs in the pathogenesis and prognosis of different types of cancer such as cancers of the oral cavity, esophagus, skin, kidney, prostate, thyroid, liver, lung, colon, and cervix (64-68). In addition, hBDs play an important role in promoting or inhibiting cancer cell proliferation/migration depending on the origin and type of the cancer cell, and 
the outcome may be associated with expression levels of hBDs in the tumor $(66,67)$. A literature review revealed that the field is rife with inconsistent findings that make it difficult to ascertain the role of hBDs in neoplasia and immunity associated with BCLM $(68,69)$. Still, this pilot study warrants a larger analytical study at transcriptomic and post-transcriptomic levels to confirm the findings. However, this warrants further comprehensive investigation in the future with comprehensive in vitro and in vivo studies on the mechanism of hBDs in BCLM cell growth suppression. The present study had some limitations, such as the small sample size, using a CTC isolation method, and the lack of comprehensive gene expression profiling between newly diagnosed and recurrent liver metastases cancer. Therefore, these data should be substantiated by appropriate prospective and comprehensive studies. Although the results provide direct data to support the prognostic potential of CTC cluster signatures in patients with BCLM, further genomic analysis of single CTCs in comparison with WBCs is required to confirm the findings.

In conclusion, we found similar clinicopathological characteristics among low-CTC and high-CTC BCLM patients. A novel finding from our study is that increased CTC numbers in BCLM patients are more closely associated with newly diagnosed liver metastasis. We also confirmed the CNV patterns of BCLM tumors with those of histologically similar tumors. Importantly, the enriched CTC-CNV pathways and genes showed a pattern comparable to newly diagnosed liver metastases, but the mutation pattern of CTCs was different from that of recurrent liver metastases. Future clinical studies with reliable and reproducible sample collection with standardized protocols are necessary to fully research the prognostic potential of CTC cluster signatures in patients with BCLM.

\section{Acknowledgements}

The authors would like to express their appreciation to the Department of Oncology, Affiliated Hospital of Southwest Medical University, Luzhou, China, Tianjin Chase Sun Pharmaceutical Co., Tianjin, China and Sichuan Provincial Center for Gynaecology and Breast Disease, Luzhou, China for their financial support. The authors thank patients who provided samples. We also wish to thank the Nuclear Medicine, Pathology and Oncology department of Southwest Medical University for their assistance in this project. Part of the project's findings has been previously presented as a poster in a conference of European Society for Medical Oncology (ESMO)-Asia 2019 (Poster number; 42P). We also gratefully acknowledge all authors and co-workers for their contributions.

\section{Funding}

The present study was supported in part by Major Cultivation Projects of Achievement Transformation in Sichuan Colleges and Universities (18CZ0043), Scientific Research Foundation for Doctors of the Affiliated Hospital of Southwest Medical University (20016), Natural Science Foundation of Tianjin (19JCQNJC12500), Project funded by China Postdoctoral Science Foundation (2019M661033) and Scientific Research Foundation for Doctors of the Affiliated Hospital of Southwest Medical University (18080).

\section{Availability of data and materials}

All the datasets generated and analyzed during the present study are available from the corresponding author on reasonable request.

\section{Authors' contributions}

$\mathrm{LiZ}$ and SI conceived the research idea and designed the study. LiZ, LG, SL and JZ searched and collected the samples and data. LeZ, QW and GC performed the radiology and pathology analyses. SI, MM, and MDS implemented the WGS, omics-data and bioinformatics analyses. LeZ and GC assisted in supervision of the research. LiZ, SI, Lez and MDS were in charge of language revision. All authors contributed to data discussion and analyses and revised the manuscript as well as reviewed the manuscript. All authors read and approved the manuscript and agree to be accountable for all aspects of the research in ensuring that the accuracy or integrity of any part of the work are appropriately investigated and resolved.

\section{Ethics approval and consent to participate}

The present study was approved by the Ethics Committee/ Institutional Review Board of the Department of Oncology, Affiliated Hospital of Southwest Medical University (Luzhou, Sichuan, China). Additionally, all patient-related procedures and protocol were approved under the guidelines of the Declaration of Helsinki. Prospective volunteers were informed in detail about the purpose and procedure of the study and a written consent form was collected from all participants before the study.

\section{Patient consent for publication}

Not applicable.

\section{Competing interests}

The authors declare that they have no competing interests.

\section{References}

1. Gerratana L, Fanotto V, Bonotto M, Bolzonello S, Minisini AM, Fasola G and Puglisi F: Pattern of metastasis and outcome in patients with breast cancer. Clin Exp Metastasis 32: 125-133, 2015.

2. Bonotto M, Gerratana L, Poletto E, Driol P, Giangreco M, Russo S, Minisini AM, Andreetta C, Mansutti M, Pisa FE, et al: Measures of outcome in metastatic breast cancer: Insights from a real-world scenario. Oncologist 19: 608-615, 2014.

3. Bale R, Putzer D and Schullian P: Local treatment of breast cancer liver metastasis. Cancers (Basel) 11: 1-15, 2019.

4. Jung SY, Sereika SM, Linkov F, Brufsky A, Weissfeld JL and Rosenzweig M: The effect of delays in treatment for breast cancer metastasis on survival. Breast Cancer Res Treat 130: 953-964, 2011.

5. Ma R, Feng Y, Lin S, Chen J, Lin H, Liang X, Zheng H and Cai X: Mechanisms involved in breast cancer liver metastasis. J Transl Med 13: 64, 2015.

6. Yoo B, Kavishwar A, Wang P, Ross A, Pantazopoulos P, Dudley M, Moore A and Medarova Z: Therapy targeted to the metastatic niche is effective in a model of stage IV breast cancer. Sci Rep 7: 45060, 2017.

7. Echeverria GV, Powell E, Seth S, Ge Z, Carugo A, Bristow C, Peoples M, Robinson F, Qiu H, Shao J, et al: High-resolution clonal mapping of multi-organ metastasis in triple negative breast cancer. Nat Commun 9: 5079, 2018. 
8. Verma S, Kalita B, Bajaj S, Prakash H, Singh AK and Gupta ML: A combination of podophyllotoxin and rutin alleviates radiation-induced pneumonitis and fibrosis through modulation of lung inflammation in mice. Front Immunol 8: 658-672, 2017.

9. Kimbung S, Loman N and Hedenfalk I: Clinical and molecular complexity of breast cancer metastases. Semin Cancer Biol 35: 85-95, 2015.

10. Liu ZJ, Semenza GL and Zhang HF: Hypoxia-inducible factor 1 and breast cancer metastasis. J Zhejiang Univ Sci B 16: 32-43, 2015.

11. Insua-Rodríguez $\mathbf{J}$ and Oskarsson $\mathrm{T}$ : The extracellular matrix in breast cancer. Adv Drug Deliv Rev 97: 41-55, 2016.

12. Obeid E, Nanda R, Fu YX and Olopade OI: The role of tumor-associated macrophages in breast cancer progression (review). Int J Oncol 43: 5-12, 2013.

13. Irshad S, Flores-Borja F, Lawler K, Monypenny J, Evans R, Male V, Gordon P, Cheung A, Gazinska P, Noor F, et al: ROR $\gamma \mathrm{t}+$ innate lymphoid cells promote lymph node metastasis breast cancers. Cancer Res 77: 1083-1096, 2017.

14. Silva S, Danson S, Teare D, Taylor F, Bradford J, McDonagh AJG, Salawu A, Wells G, Burghel GJ, Brock I, et al: Genome-wide analysis of circulating cell-free DNA copy number detects active melanoma and predicts survival. Clin Chem 64: 1338-1346, 2018.

15. Gao Y, Ni X, Guo H, Su Z, Ba Y, Tong Z, Guo Z, Yao X, Chen X, Yin J, et al: Single-cell sequencing deciphers a convergent evolution of copy number alterations from primary to circulating tumor cells. Genome Res 27: 1312-1322, 2017.

16. Poell JB, Mendeville M, Sie D, Brink A, Brakenhoff RH and Ylstra B: ACE: Absolute copy number estimation from low-coverage whole-genome sequencing data. Bioinformatics 35: 2847-2849, 2019

17. Xiao YB, Zhang B and Wu YL: Radiofrequency ablation versus hepatic resection for breast cancer liver metastasis: A systematic review and meta-analysis. J Zhejiang Univ Sci B 19: 829-843, 2018.

18. Treska V, Cerna M, Kydlicek T and Treskova I: Prognostic factors of breast cancer liver metastasis surgery. Arch Med Sci 11: 683-685, 2015 .

19. Mego M, Karaba M, Minarik G, Benca J, Silvia J, Sedlackova T, Manasova D, Kalavska K, Pindak D, Cristofanilli M, et al: Circulating tumor cells with epithelial-to-mesenchymal transition phenotypes associated with inferior outcomes in primary breast cancer. Anticancer Res 39: 1829-1837, 2019.

20. Arkadius P, Volkmar M, Jens H, Wolfgang J and Tanja F: Circulating tumor cells in metastatic breast cancer: Clinical relevance and biological potential. Curr Opin Obstet Gynecol 31 76-81, 2019.

21. Mansouri S, Mokhtari-Hesari P, Naghavi-Al-Hosseini F, Majidzadeh-A K and Farahmand L: The prognostic value of circulating tumor cells in primary breast cancer prior to any systematic therapy: A systematic review. Curr Stem Cell Res Ther 14: 519-529, 2019.

22. Loh J, Jovanovic L, Lehman M, Capp A, Pryor D, Harris M, Nelson C and Martin J: Circulating tumor cell detection in high-risk non-metastatic prostate cancer. J Cancer Res Clin Oncol 140: 2157-2162, 2014.

23. Wang Y, Liu Y, Zhang L, Tong L, Gao Y, Hu F, Lin PP, Li B and Zhang T: Vimentin expression in circulating tumor cells (CTCs) associated with liver metastases predicts poor progression-free survival in patients with advanced lung cancer. J Cancer Res Clin Oncol 145: 2911-2920, 2019.

24. Duffy MJ, McDermott EW and Crown J: Blood-based biomarkers in breast cancer: From proteins to circulating tumor cells to circulating tumor DNA. Tumour Biol 40: 1010428318776169, 2018.

25. Cristofanilli M, Pierga JY, Reuben J, Rademaker A, Davis AA, Peeters DJ, Fehm T, Nolé F, Gisbert-Criado R, Mavroudis D, et al: The clinical use of circulating tumor cells (CTCs) enumeration for staging of metastatic breast cancer (MBC): International expert consensus paper. Crit Rev Oncol Hematol 134: 39-45, 2019.

26. Bai L, Du Y, Peng J, Liu Y, Wang Y, Yang Y and Wang C: Peptide-based isolation of circulating tumor cells by magnetic nanoparticles. J Mater Chem B Mater Biol Med 2: 4080-4088, 2014.

27. Bailey PC and Martin SS: Insights on CTC biology and clinical impact emerging from advances in capture technology. Cells 8: 553,2019

28. Imani S, Cheng J, Mobasher-Jannat A, Wei C, Fu S, Yang L, Jadidi K, Khosravi MH, Mohazzab-Torabi S, Shasaltaneh MD, et al: Identification of a novel RPGRIP1 mutation in an Iranian family with leber congenital amaurosis by exome sequencing. J Cell Mol Med 22: 1733-1742, 2018.
29. Zong C, Lu S, Chapman AR and Xie XS: Genome-wide detection of single-nucleotide and copy-number variations of a single human cell. Science 338: 1622-1626, 2012.

30. Kent WJ, Sugnet CW, Furey TS, Roskin KM, Pringle TH, Zahler AM and Haussler D: The human genome browser at UCSC. Genome Res 12: 996-1006, 2002.

31. Samur MK: RTCGAToolbox: A new tool for exporting TCGA firehose data. PLoS One 9: e106397, 2014.

32. Silva GO, Siegel MB, Mose LE, Parker JS, Sun W, Perou CM and Chen M: SynthEx: A synthetic-normal-based DNA sequencing tool for copy number alteration detection and tumor heterogeneity profiling. Genome Biol 18: 66, 2017.

33. Seiser EL and Innocenti F: Hidden markov model-based CNV detection algorithms for illumina genotyping microarrays Cancer Inform 13 (Suppl 7): 77-83, 2015.

34. Manzo A, Montanino A, Carillio G, Costanzo R, Sandomenico C, Normanno N, Piccirillo MC, Daniele G, Perrone F, Rocco G and Morabito A: Angiogenesis inhibitors in NSCLC. Int J Mol Sci 18: 2021, 2017.

35. Subramanian A, Tamayo P, Mootha VK, Mukherjee S, Ebert BL, Gillette MA, Paulovich A, Pomeroy SL, Golub TR, Lander ES and Mesirov JP: Gene set enrichment analysis: A knowledge-based approach for interpreting genome-wide expression profiles. Proc Natl Acad Sci USA 102: 15545-15550, 2005.

36. Mootha VK, Lindgren CM, Eriksson KF, Subramanian A Sihag S, Lehar J, Puigserver P, Carlsson E, Ridderstråle M, Laurila E, et al: PGC-1alpha-responsive genes involved in oxidative phosphorylation are coordinately downregulated in human diabetes. Nat Genet 34: 267-273, 2003

37. Liu Y, Liu J, Lu J, Peng J, Juan L, Zhu X, Li B and Wang Y: Joint detection of copy number variations in parent-offspring trios. Bioinformatics 32: 1130-1137, 2016.

38. Lu X, Ye K, Zou K and Chen J: Identification of copy number variation-driven genes for liver cancer via bioinformatics analysis. Oncol Rep 32: 1845-1852, 2014.

39. Williams A, Balic M, Datar R and Cote R: Size-based enrichment technologies for CTC detection and characterization. Recent Results Cancer Res 195: 87-95, 2012.

40. Che J, Yu V, Garon EB, Goldman JW and Di Carlo D: Biophysical isolation and identification of circulating tumor cells. Lab Chip 17: 1452-1461, 2017.

41. Krebs MG, Sloane R, Priest L, Lancashire L, Hou JM, Greystoke A, Ward TH, Ferraldeschi R, Hughes A, Clack G, et al: Evaluation and prognostic significance of circulating tumor cells in patients with non-small-cell lung cancer. J Clin Oncol 29: 1556-1563, 2011.

42. van den Bos H, Bakker B, Spierings DCJ, Lansdorp PM and Foijer F: Single-cell sequencing to quantify genomic integrity in cancer. Int J Biochem Cell Biol 94: 146-150, 2018.

43. Khetrapal P, Lee MWL, Tan WS, Dong L, de Winter P, Feber A and Kelly JD: The role of circulating tumour cells and nucleic acids in blood for the detection of bladder cancer: A systematic review. Cancer Treat Rev 66: 56-63, 2018.

44. Payne K, Brooks J, Spruce R, Batis N, Taylor G, Nankivell P and Mehanna $\mathrm{H}$ : Circulating tumour cell biomarkers in head and neck cancer: Current progress and future prospects. Cancers (Basel) 11: 1115, 2019.

45. Ortiz V and Yu M: Analyzing circulating tumor cells one at a time. Trends Cell Biol 28: 764-775, 2018.

46. Liu X, Wang J and Chen L: Whole-exome sequencing reveals recurrent somatic mutation networks in cancer. Cancer Lett 340: 270-276, 2013.

47. Ren T, Suo J, Liu S, Wang S, Shu S, Xiang Y and Lang JH: Using low-coverage whole genome sequencing technique to analyze the chromosomal copy number alterations in the exfoliative cells of cervical cancer. J Gynecol Oncol 29: e78, 2018.

48. Brouwer A, De Laere B, Peeters D, Peeters M, Salgado R, Dirix L and Van Laere S: Evaluation and consequences of heterogeneity in the circulating tumor cell compartment. Oncotarget 7: 48625-48643, 2016

49. Huang W, Skanderup AJ and Lee CG: Advances in genomic hepatocellular carcinoma research. Gigascience 7: 1-13, 2018

50. Scatena R, Bottoni P and Giardina B: Circulating tumour cells and cancer stem cells: A role for proteomics in defining the interrelationships between function, phenotype and differentiation with potential clinical applications. Biochim Biophys Acta 1835: 129-143, 2013.

51. Salvianti F and Pinzani P: The diagnostic potential of mutation detection from single circulating tumor cells in cancer patients. Expert Rev Mol Diagn 17: 975-981, 2017. 
52. Salvianti F, Pazzagli M and Pinzani P: Single circulating tumor cell sequencing as an advanced tool in cancer management. Expert Rev Mol Diagn 16: 51-63, 2016.

53. Mohtar MA, Syafruddin SE, Nasir SN and Low TY: Revisiting the roles of pro-metastatic EpCAM in cancer. Biomolecules 10: $255,2020$.

54. de Wit $S$, van Dalum G, Lenferink AT, Tibbe AG, Hiltermann TJ, Groen HJ, van Rijn CJ and Terstappen LW: The detection of $\operatorname{EpCAM}(+)$ and $\operatorname{EpCAM(-)~circulating~tumor~cells.~Sci~Rep~5:~}$ 12270, 2015.

55. Adams DL, Martin SS, Alpaugh RK, Charpentier M, Tsai S, Bergan RC, Ogden IM, Catalona W, Chumsri S, Tang CM and Cristofanilli M: Circulating giant macrophages as a potential biomarker of solid tumors. Proc Natl Acad Sci USA 111: 3514-3519, 2014.

56. Gires O and Stoecklein NH: Dynamic EpCAM expression on circulating and disseminating tumor cells: Causes and consequences. Cell Mol Life Sci 71: 4393-4402, 2014.

57. Grover PK, Cummins AG, Price TJ, Roberts-Thomson IC and Hardingham JE: Circulating tumour cells: The evolving concept and the inadequacy of their enrichment by EpCAM-based methodology for basic and clinical cancer research. Ann Oncol 25: 1506-1516, 2014

58. Mikolajczyk SD, Millar LS, Tsinberg P, Coutts SM, Zomorrodi M, Pham T, Bischoff FZ and Pircher TJ: Detection of EpCAM-negative and cytokeratin-negative circulating tumor cells in peripheral blood. J Oncol 2011: 252361, 2011.

59. Yokobori T, Iinuma H, Shimamura T, Imoto S, Sugimachi K, Ishii H, Iwatsuki M, Ota D, Ohkuma M, Iwaya T, et al: Plastin3 is a novel marker for circulating tumor cells undergoing the epithelial-mesenchymal transition and is associated with colorectal cancer prognosis. Cancer Res 73: 2059-2069, 2013.

60. Auman JT and McLeod HL: Colorectal cancer cell lines lack the molecular heterogeneity of clinical colorectal tumors. Clin Colorectal Cancer 9: 40-47, 2010.

61. Ghosh SK, McCormick TS and Weinberg A: Human Beta defensins and cancer: Contradictions and common ground. Front Oncol 9: 341-354, 2019.
62. Xu Z, Xu P, Fan W, Huang B, Cheng Q, Zhang Z, Wang P and Yu M: The effect of an alternative chromosome 17 probe on fluorescence in situ hybridization for the assessment of HER2 amplification in invasive breast cancer. Exp Ther Med 18: 2095-2103, 2019.

63. Zubenko OS, Semeniuk DO, Starenka IO and Pogribnyy PV: Effect of cytostatic agents on expression levels of human beta-defensins-1-4 in A431 and MCF-7 cell lines. Exp Oncol 40: 79-81, 2018.

64. Shestakova T,Zhuravel E, Bolgova L,Alekseenko O, Soldatkina M and Pogrebnoy P: Expression of human beta-defensins-1, 2 and 4 mRNA in human lung tumor tissue: A pilot study. Exp Oncol 30: 153-156, 2008

65. Joly S, Compton LM, Pujol C, Kurago ZB and Guthmiller JM: Loss of human beta-defensin 1,2 , and 3 expression in oral squamous cell carcinoma. Oral Microbiol Immunol 24: 353-360, 2009.

66. Prahl A, Pazgier M, Alexandratos J and Lubkowski J: Human $\beta$-defensin 4 - defensin without the 'twist'. Postepy Biochem 62: 349-361, 2016.

67. Markeeva N, Lisovskiy I, Lyzogubov V, Usenko V, Soldatkina M, Merentsev S, Zaitsev S, Kondratskii Y, Tofan A, Osinskiy S, et al: Expression of beta-defensin-2 in human gastric tumors: A pilot study. Exp Oncol 27: 30-135, 2005.

68. Kida-Takaoka S, Yamaai T, Mizukawa N, Murakami J and Iida S Surrounding cells affect the gene expression pattern of human beta-defensins in squamous cell carcinoma in vitro. Anticancer Res 34: 6443-6449, 2014.

69. Winter J, Kraus D, Reckenbeil J and Probstmeier R: Oncogenic relevant defensins: Expression pattern and proliferation characteristics of human tumor cell lines. Tumour Biol 37: 7959-7966, 2016.

This work is licensed under a Creative Commons Attribution-NonCommercial-NoDerivatives 4.0 International (CC BY-NC-ND 4.0) License. 